\title{
Transient Detections and Other Real-Time Data Processing from MASTER-VWF Wide-Field Cameras
}

\author{
Evgeny Gorbovskoy, ${ }^{1}$ Kirill Ivanov, ${ }^{2}$ Vladimir Lipunov, ${ }^{1}$ Victor Kornilov, ${ }^{1}$ \\ Alexander Belinski, ${ }^{1}$ Nikolaj Shatskij, ${ }^{1}$ Dmitry Kuvshinov, ${ }^{1}$ Nataly Tyurina, ${ }^{1}$ \\ Pavel Balanutsa, ${ }^{1}$ Vadim Chazov, ${ }^{1}$ Artem Kuznetsov, ${ }^{1}$ Petr Kortunov, ${ }^{1}$ Andrey Tlatov, ${ }^{3}$ \\ Alexander Parkhomenko, ${ }^{3}$ Vadim Krushinsky, ${ }^{4}$ Ivan Zalozhnyh, ${ }^{4}$ Alexander Popov, ${ }^{4}$ \\ Taisia Kopytova, ${ }^{4}$ Sergey Yazev, ${ }^{2}$ and Alexander Krylov ${ }^{1}$
}

${ }^{1}$ Sternberg Astronomical Institute, Moscow State University 13 Univeristetskij pr-t, Moscow 119991, Russia

${ }^{2}$ Irkutsk State University, 1 Karl Marks street, Irkutsk 664003, Russia

${ }^{3}$ Kislovodsk Solar Station, P.O. Box 145, 100 Gagarina street, Kislovodsk 357700, Russia

${ }^{4}$ Ural State University, 51 Lenina pr-t, Ekaterinburg 620083, Russia

Correspondence should be addressed to Evgeny Gorbovskoy, gorbovskoy@gmail.com

Received 2 July 2009; Revised 8 October 2009; Accepted 10 March 2010

Academic Editor: Joshua S. Bloom

Copyright (C) 2010 Evgeny Gorbovskoy et al. This is an open access article distributed under the Creative Commons Attribution License, which permits unrestricted use, distribution, and reproduction in any medium, provided the original work is properly cited.

Construction of robotic observatories has developed into an important and thriving field of astronomy. Their large field of view combined with the capability to be pointed at any direction makes robotic astronomical systems indispensable for tasks involving searches for transients like GRB, supernovae explosions, novae, and so forth, where both the time and direction of the search are impossible to predict. This paper describes prompt GRB observations made with MASTER-VWF wide-field cameras and the methods of image analysis and classification of transients used for real-time data processing. During seven months of operation six synchronous observations of gamma-ray bursts have been made using MASTER VWF facilities deployed in Kislovodsk and Irkutsk. In all cases high upper limits have been obtained.

\section{Introduction}

1.1. Very Wide-Field Telescopes. Currently, several dozen robotic telescopes are operated worldwide, each of them usually meant for certain dedicated tasks. A special case of these instruments is superWide-Field telescopes with fields of view ranging from hundreds to thousands square degrees. It is impossible to make a very Wide-Field system with a large aperture using now available CCDs and optical systems, and that is why the apertures of very WideField cameras are limited to $5-15 \mathrm{~cm}$. All very Wide-Field cameras are designed to record various optical transients. Most of the optical transients are of circumterraneous origin meteors and satellites; however, very wide-field cameras may also provide valuable data on the prompt emission of cosmological GRBs. A striking example is the incredibly successful observation of GRB080319B that was performed within the framework of the Russian-Italian experiment TORTORA [1] and Polish experiment "Pi of the Sky" [2]. All transients are highly variable and therefore snapshots must be taken at intervals of several seconds or even less. Hence solutions capable of operating without dead time (such as the time needed for CCD readout), like MASTER-VWF [3], TORTORA, and others (see Table 1), have an important advantage over other systems.

The operation of any automatic astronomical facility is supported by a dedicated software package, which also performs primary image processing and data storage. Which software to use for an automatic astronomical system depends on the particular task the facility is meant to perform, and multipurpose solutions are therefore unsuitable in such cases. Classical universal techniques are even less suited for superwide-Field observations, because of the problems due to the field curvature and very large data amount. 
TABle 1: Comparative characteristics of super Wide-Field systems.

\begin{tabular}{lccc}
\hline Project & FOV & Exposure [s] & Limiting magnitude $[\mathrm{Mag}(\mathrm{V})]$ \\
\hline WIDGET [4] & $44^{\circ} \times 44^{\circ} \times 3$ & 5 & $10^{m}$ \\
RAPTOR & $40^{\circ} \times 40^{\circ}$ & 60 & $12^{m}$ \\
Pi of the Sky [2] & $22^{\circ} \times 22^{\circ}$ & 10 & $11.5^{m}$ \\
Yatsugatake Cameras & $85^{\circ} \times 70^{\circ}$ & 8 & $5^{m}$ \\
FAVOR [5] & $16^{\circ} \times 24^{\circ}$ & 0.13 & $11.5^{m}$ \\
TORTORA [6] & $24^{\circ} \times 32^{\circ}$ & 0.13 & $10.5^{m}$ \\
\hline MASTER-VWF (1) [3] & $18^{\circ} \times 25^{\circ}$ or $28^{\circ} \times 45^{\circ}$ & $0.3-10$ & $12^{m}(5-\mathrm{s}) 9.5^{m}(0.3 \mathrm{~s})$ \\
MASTER-VWF (4) [7] & $4 \times 28^{\circ} \times 42^{\circ}$ & $0.3-10$ & $11.5^{m}(5-\mathrm{s}) 9.5^{m}(0.3 \mathrm{~s})$ \\
\hline
\end{tabular}

1.2. MASTER-Mobile Astronomical System of the Telescope Robot. MASTER was the first robotic telescope of this kind constructed in Russia. It was built and tested by students, postgraduate students, and lecturers of SAI MSU in 2002 (http://observ.pereplet.ru). MASTER has evolved into a broad network of telescopes spread across the entire country. A detailed description of the MASTER facility can be found in [7]. Below we discuss the basic properties of MASTER wield field cameras (MASTER-VWF).

The main task of the MASTER-VWF experiment is to perform continuous all-sky monitoring with the aim to detect all objects absent in the available astronomical catalogues. This includes, in particular the folowing:

(i) prompt detection of GRB emission synchronously with gamma-ray space observatories,

(ii) search for star-like transients of unknown origin and orphan bursts,

(iii) detection of meteors and determination of their basic parameters: brightness (photometry), velocity (astrometry), and altitude of combustion in the atmosphere (triangulation),

(iv) detection of satellites and space debris and determination of their basic parameters-astrometry, velocity, brightness, and altitude above the earth's surface.

For these purposes, a completely robotic MASTERVWF4 facility with four wide-field cameras and a total field of view of 4000 square degrees ( $\mathrm{deg}^{2}$ below) has been deployed at the MSU Caucasian Mountain Astronomical Observatory near Kislovodsk $(2075 \mathrm{~m})$. Another facility-MASTER-VWF2 (two cameras and $2000 \mathrm{deg}^{2}$ field of view)-has been deployed near Irkutsk. In Kislovodsk cameras are set on parallactic mounts in pairs 702 meters apart (see Figure 2). Each mount has an automated dome and two fast and powerful (11 Megapixels) Prosilica GE 4000 CCD cameras with Nikkor $50 \mathrm{~mm}(f / 1.2)$ lenses. The camera is capable of continuous imaging with a speed of one frame in 0.2 to 60 seconds with no time gaps. The $702 \mathrm{~m}$ baseline allows the facility in Kislovodsk to determine the parallaxes of circumterraneous objects and reconstruct their altitudes from the results of astrometric reduction.

All telescopes operate in a fully autonomous mode: at night, if the weather conditions permit (a "Boltwood Cloud Sensor" provides the current weather report updated every three seconds), the cameras begin to automatically survey the sky. The frames taken are then processed and stored in a special data storage device. Because of the very large data flow (up to $700 \mathrm{~Gb}$ per night), the images can be kept only for several days and then most of them have to be erased, except for some images with objects of special interest like meteors, candidate optical transients, and satellites. We also keep the images taken simultaneously with GRBs. Nevertheless we save the coadd images, with total exposure from a minute and more. Thereby we form archive, which soon will be available to the community. ${ }^{1}$ The survey is halted and the telescope dome is closed every time when the weather deteriorates or at dawn. The operation of the entire MASTER-VWF4 facility is controlled by seven computers (see Figure 3): four computers to control the operation of the Prosilica GE 4000 CCD camera and perform source extraction, two computers to control the operation of the dome and mount, and one big quad-core server to process and store the data obtained and publish it in the Web.

The majority developed in the project software products are grouped in independent applications (astrometry, transient events classification, batch pipline data processing) which at certain upgrade can be used and in other projects, operations on such upgrade are carried on.

The facility is a complex engineering system, which needs sophisticated and powerful software to control it, perform observations and data processing. The data flow can be as high as several Terabytes/day (in the case of very short exposures on the order of $0.2 \mathrm{~s}$ ), requiring highly optimized software, powerful data storage facilities, and interfaces. With 5 -second long exposures the limiting is $11.5-12$ on each frame depending on weather conditions. Thus every image contains about 10-15 thousand objects, resulting in a data flow that is impossible to store and has to be processed in real-time.

A special software package has been developed for this purpose. It allows the coordinates of all objects and their photometric characteristics (profile, magnitude) to be determined in real-time, and is also capable of finding and analysing optical transients. MASTER-VWF can measure the coordinates of the objects with an error smaller than 10 arcseconds. The system is supplied with a daily updated database of the ephemerides of artificial satellites and a program developed to identify the satellites in real-time. The program is capable of finding new artificial satellites, to 


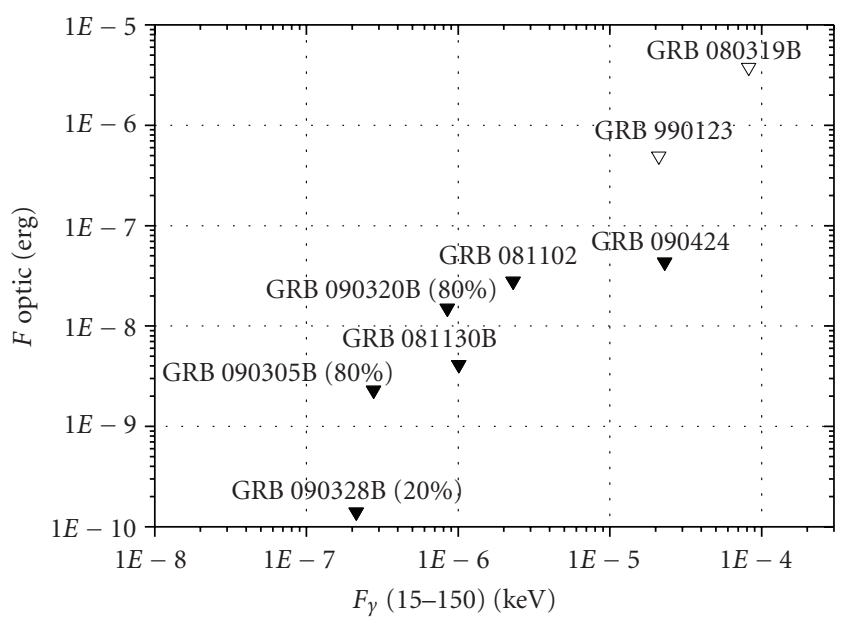

(a)

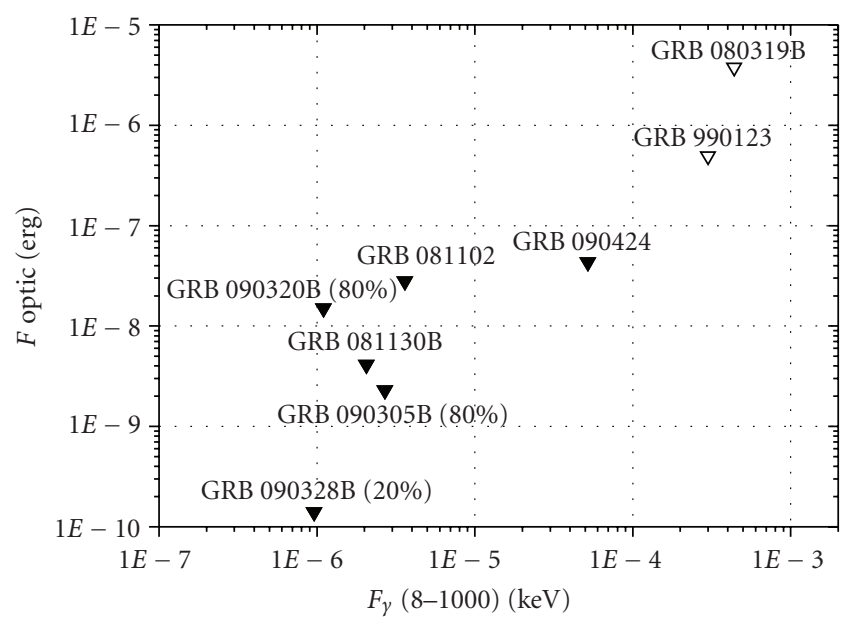

(b)

FIGURE 1: Summary results of synchronous observations of a gamma-ray burst: dependence plotted as a function of gamma-ray fluence in the soft $(15-150 \mathrm{keV})$ and hard $(8-1000 \mathrm{keV})$ energy interval.

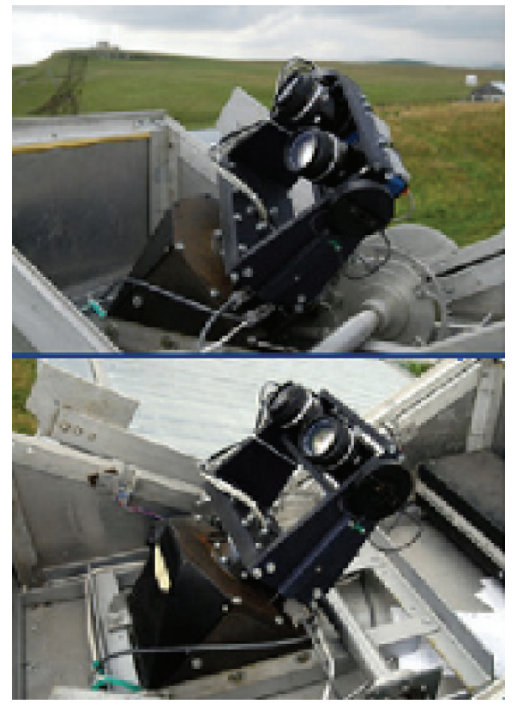

(a)

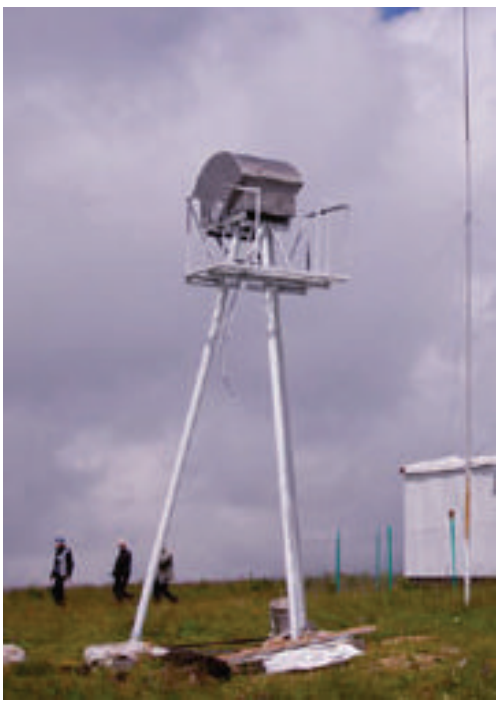

(b)

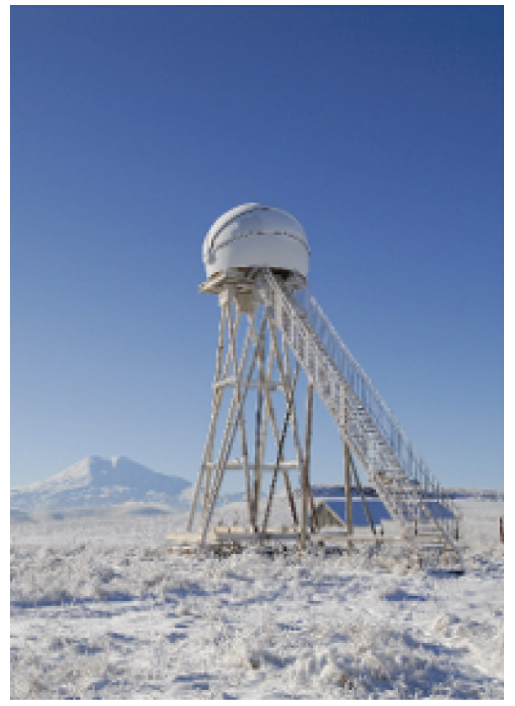

(c)

FIgURE 2: The equipment of the MASTER facility deployed at the MSU Caucasian Mountain Astronomical Observatory near Kislovodsk. From the left to right: (a) the East and West cameras of the MASTER-VWF4 northern installation, (b) the main tower and shelter of the southern MASTER-VWF4 installation, and (c) the main tower of the MASTER-2 telescope [7].

observe the destruction of known artificial satellites and not only to control space debris but also observe the process of its formation. Sometimes unknown or lost objects are recorded. For example, on October 20, 2008 MASTER-VWF recorded the debris of the American military satellite USA114Deb (see Figure 4).

A total of six synchronous observations of gamma-ray bursts have been made by MASTER-VWF in Kislovodsk and Irkutsk during seven months of their operation. In all cases high upper limits have been determined (see Table 2 and Figure 1).

In the following sections we describe these and other results in more detail.

\section{Primary Reduction in Wide-Fields: Astrometry and Photometry}

Let us consider the methods of astromeric and photometric reduction of a Wide-Field in more detail. The identification of the field of view and calculation of the coordinate grid for the image is based on the table of Cartesian imageplane coordinates $(X, Y)$ produced by program used for the extraction of objects (SExtractor) and the Tycho-2 astrometric catalogue, which contains exact coordinates for most of the stars down to a limiting magnitude of $11^{\mathrm{m}}$. 5. To perform this task, the program should meet high requirements in terms of speed of processing (10-15 seconds 


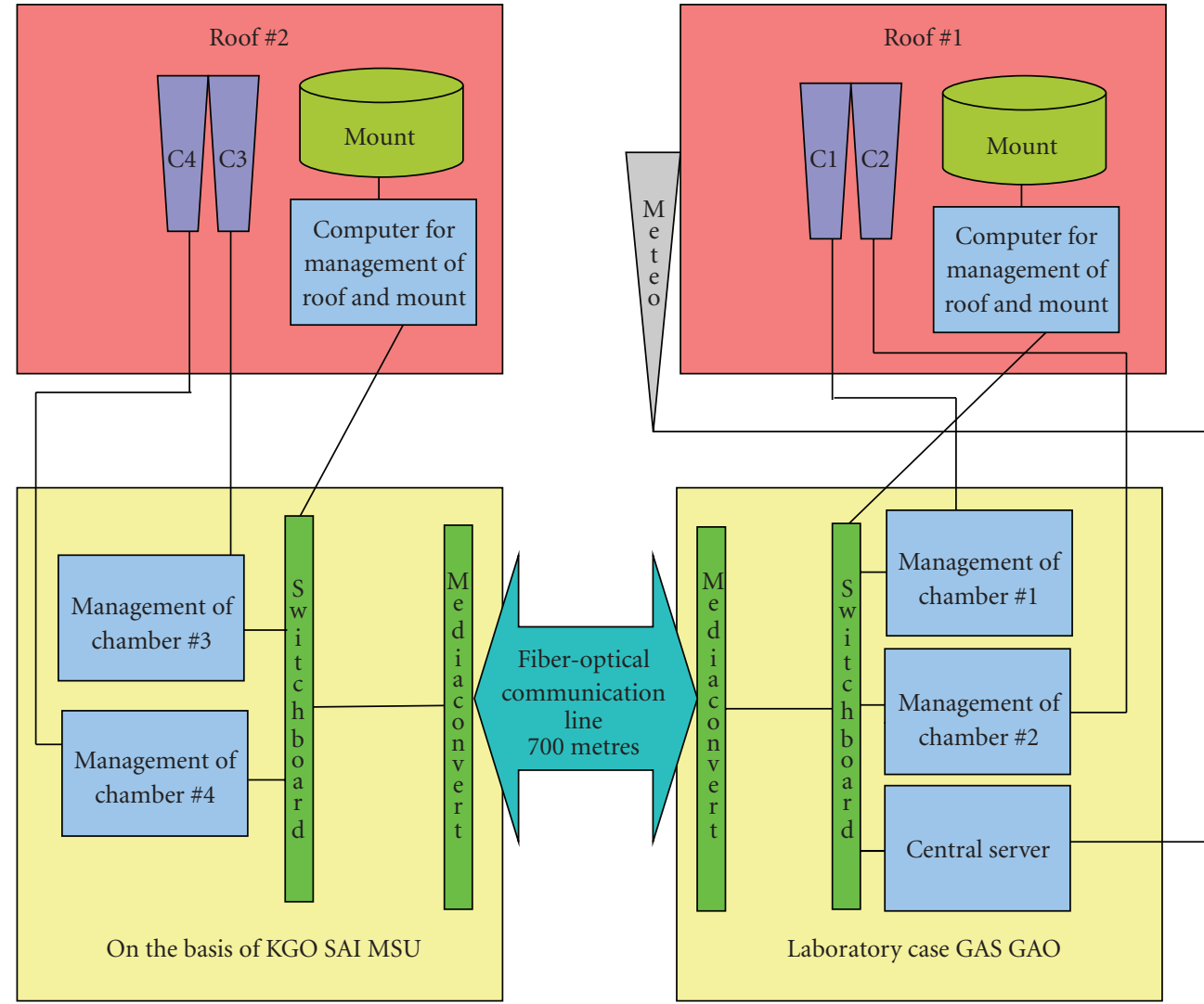

FIGURE 3: Schematic diagram of the layout of the equipment in the MASTER-VWF4 experiment.

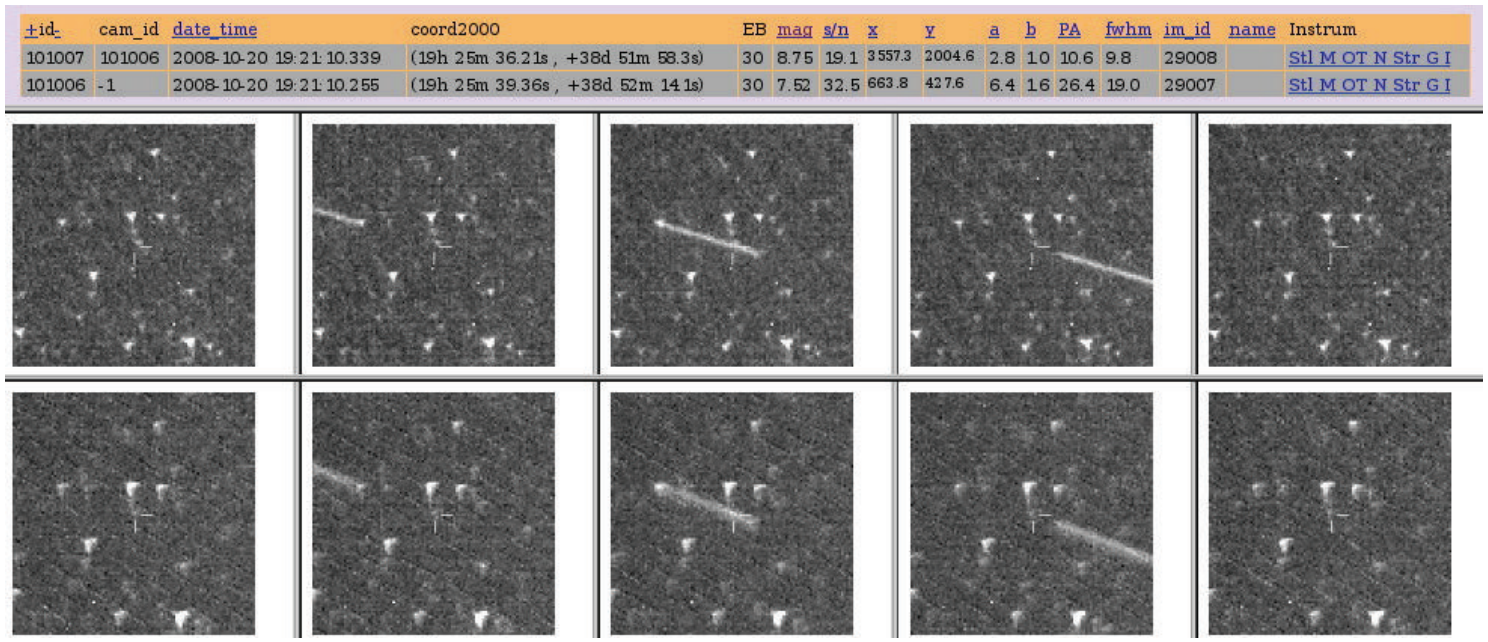

FIgure 4: Debris of the American military satellite USA114Deb found via the web interface of the MASTER system. The top and bottom rows of the images are taken by the Northern and Southern cameras, respectively. The parallactic shift is apparent even to an unaided eye. The measured parallax implies a height of $4000 \pm 200$ kilometers.

and less) for a field containing about 10000 stars, ensure high probability of identification (more 99\%), be applicable to various fields ranging in size from narrow (tens of square minutes) to superwide (thousand $\mathrm{deg}^{2}$ ) fields, allow for a certain freedom in the telescope parameters, because the focal length and other parameters may change in the course of the year due to the variation of the outside temperature, and be capable of operating with approximate initial midfield coordinates provided that they are within the field of view.

All processing in MASTER experiments is performed under Linux OS and hence no ready-made software was likely to be found. The current implementation of the 
TABle 2: Prompt grb observations.

\begin{tabular}{|c|c|c|c|c|c|c|}
\hline GRB & Satellite & Site & $V$ & Optical fluence & $\begin{array}{c}\text { Gamma-ray fluence } \\
(15-150 \mathrm{keV})\end{array}$ & $\begin{array}{c}\text { Gamma-ray fluence } \\
\quad(8-1000 \mathrm{keV})\end{array}$ \\
\hline GRB990123 & BeppoSAX Konus-Wind & ROTSE-I & $8.9^{m}$ & $4.9 \cdot 10^{-7}$ & $2.1 \cdot 10^{-5}$ & $3.0 \cdot 10^{-4}$ \\
\hline GRB080319B & Swift & Pi-of-the-sky TORTORA & $5.3^{m}$ & $3.7 \cdot 10^{-6}$ & $8.3 \cdot 10^{-5}$ & $4.4 \cdot 10^{-4}$ \\
\hline GRB081102 & Swift & MASTER Kislovodsk & $<13.0^{m}$ & $<2.8 \cdot 10^{-8}$ & $2.3 \cdot 10^{-6}$ & $3.6 \cdot 10^{-6}$ \\
\hline GRB090424 & Swift & MASTER Irkursk & $<9.7^{m}$ & $<4.3 \cdot 10^{-8}$ & $2.3 \cdot 10^{-5}$ & $5.2 \cdot 10^{-5}$ \\
\hline GRB0901130B & FERMI & MASTER Kislovodsk & $<11.0^{m}$ & $<4.1 \cdot 10^{-9}$ & $1.0 \cdot 10^{-6}$ & $2.0 \cdot 10^{-6}$ \\
\hline GRB090305B & FERMI & MASTER & $<9.5^{m}$ & $<2.3 \cdot 10^{-9}$ & $2.7 \cdot 10^{-7}$ & $2.7 \cdot 10^{-6}$ \\
\hline (80\% part) & & Kislovodsk & & & & \\
\hline GRB090320B & FERMI & MASTER & $<11.0^{m}$ & $<1.5 \cdot 10^{-8}$ & $8.5 \cdot 10^{-7}$ & $1.1 \cdot 10^{-6}$ \\
\hline (80\% part) & & Kislovodsk & & & & \\
\hline GRB090328B & FERMI & MASTER & $<9.1^{m}$ & $<1.4 \cdot 10^{-10}$ & $2.1 \cdot 10^{-7}$ & $9.6 \cdot 10^{-7}$ \\
\hline (20\% part) & & Irkursk & & & & \\
\hline
\end{tabular}

"astrom"2 program is based on the algorithm of pairing similar triangles of bright stars in the image and in the catalogue. This program works perfectly with the most different images from those taken under poor weather conditions and containing a few dozen stars (typical situation for alert observations) to images in the Milky-Way fields taken during a good observing night and containing up to 100000 objects. The root-mean-square error of the measured coordinates ranges from $1 / 4$ of arcsecond (scale $2.1^{\prime \prime} /$ pixel) for most of the images taken with the basic telescope of the MASTER system to about 7-8 arcseconds for very wide-field cameras (scale 36"/pixel) (see Figure 2).

The system may also operate in a "fast" astrometry mode to process consecutive frames of the same area taken with wide-field cameras. In this mode the software uses a priori information about the field of view based on the previous frames, allowing the operating time to be reduced to less than 0.2 second. There is also a dedicated mode for more accurate determination of the coordinates of special objects (gammaray bursts, supernovae, asteroids, meteors, and others) with the local coordinate adjustment computed only in the field of the object searched using 10-25 nearest stars with wellaccurate coordinates. Our experience shows that this mode provides a factor of two improvement of the accuracy of the inferred coordinates.

Our program for extracting objects from images is based on the SExtractor software package written for the TERAPIX [8] project. SExtractor is written in the C language and offers sufficiently high performance. We adapted this program to the task of reducing images taken with MASTER, and describe our version of the program in the following chapter. The main parameter used by the extraction program is the threshold signal-to-noise ratio. This value directly affects both the program execution time and the accuracy of the results obtained. We adopt $S / N=2$ for primary selection, whereas at the subsequent stages of processing we select from the initial list only the objects with higher $S / N$ values.

2.1. Photometric Calibration. Practically all wide-field systems operate without filters. MASTER-VWF is not an exception. This poses the problem of the calibration of the photometric band of the measurements. It is clear that our instrumental photometric band is limited to a certain extent by the CCD sensitivity curve and lens transmission curve. Our task is therefore to determine instrumental magnitudes based on the magnitudes of the reference stars adopted from photometric catalogues.

To this aim, colour-colour diagrams are analysed, which relate the difference between the instrumental magnitude and the magnitude in any of the modelled bands (or combinations thereof) to a chosen colour index for each detected star. The closer is the modelled band to the instrumental band, the more horizontal appears the cloud of points on the diagram. Figure 6 shows the corresponding colourcolour diagrams for the MASTER-VWF-4 system consisting (in its photometric part) of a Nikkor $50 \mathrm{~mm} \mathrm{f/1.4} \mathrm{lens}$ and Prosilica GE4000 CCD. The instrumental photometric band can be described fairly well by the $V_{\text {TYCHO2 }}$ filter. The absolute accuracy of photometric measurements made with wide-field cameras is, on the average, of about $\Delta m_{\mathrm{abs}} \sim$ $0.25-0.35^{m}$. Note that the relative accuracy of measurements is substantially better with errors as low as $\Delta m_{\text {rel }} 3-5 \%$.

\subsection{Automatic Determination of the Limiting Magnitude of an} Image. Automatic determination of the limiting magnitude for the images taken is an important part of the operation of modern automatic sky surveys. An automatic survey must naturally include automated quality control. The automatically determined limiting magnitude may serve as some kind of objective quality assessment for the images obtained. Furthermore, the determination of the limiting magnitude is also needed for gamma-ray burst observations.

The task of the determination of the limiting magnitude can be addressed formally by setting the limiting magnitude of the frame equal to the magnitude of the faintest object. This approach; however, has a number of shortcomings. First, when the system is operated with a low extraction threshold, most of the faintest objects are noise artifacts. If the sky is covered with clouds, no real faint objects can be seen and practically all objects at the visibility limit are chance fluctuations of cloud brightness. Moreover, because of the large number of such false objects some of them 


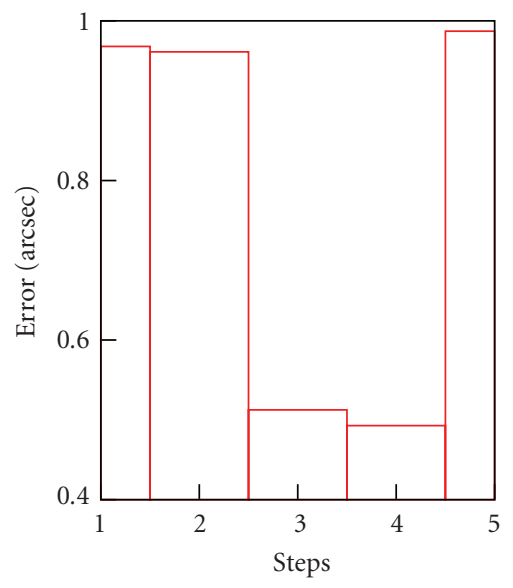

(a)

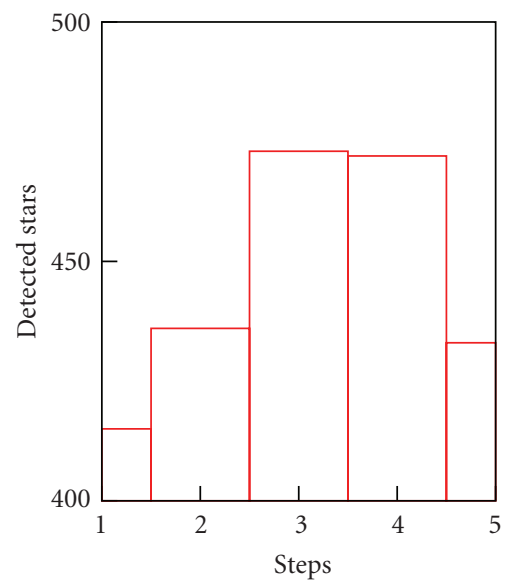

(d)

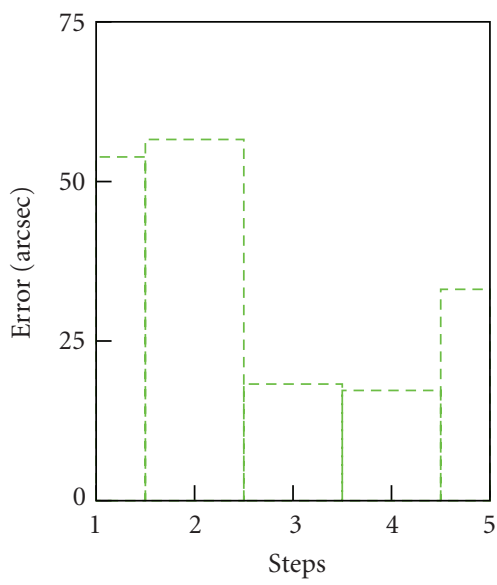

(b)

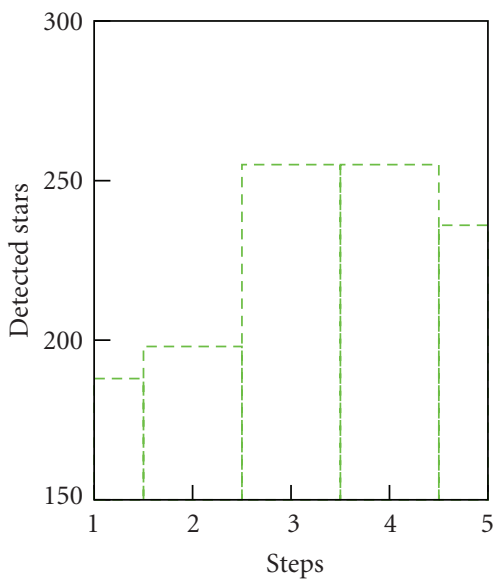

(e)

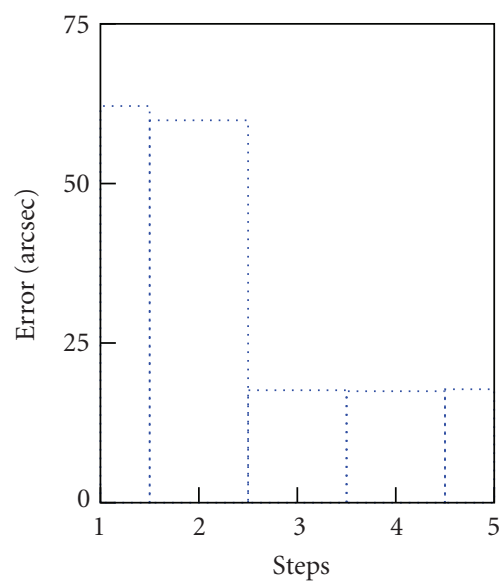

(c)

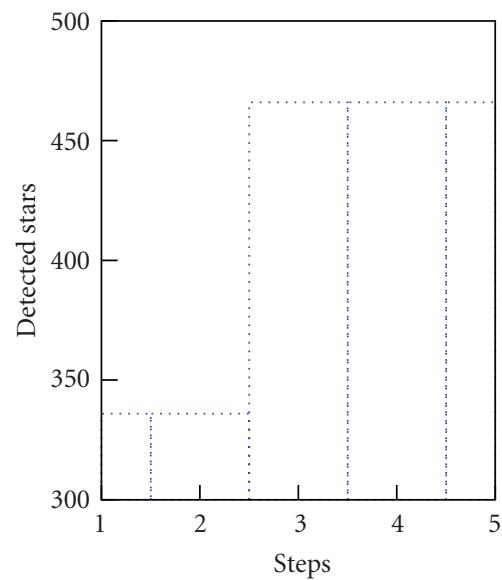

(f)

Figure 5: Average error (a, b, c) and the number of identified stars (d, e, f) as functions of the degree of the polynomial used. The dependences are shown for three different optical systems. Red (a) and (d) Rigter-Slefogt (MASTER) with a field of view of 6 deg ${ }^{2}$, Nikkor $50 \mathrm{~mm} \mathrm{f/1.2} \mathrm{(b)} \mathrm{and} \mathrm{(e)} \mathrm{with} \mathrm{a} \mathrm{field} \mathrm{of} \mathrm{view} \mathrm{of} 1000 \mathrm{deg}^{2}$, and Nikkor $50 \mathrm{~mm} \mathrm{f/1.4}$ (c) and (f) with a field of view of $1000 \mathrm{deg}^{2}$. The histograms are based on arbitrary frames from various sky areas. A third-to-fourth-degree polynomial approximation can be seen to provide optimum solution (95-99 percent of stars are identified with the minimum average error). A more detailed analysis shows that the thirddegree fit yields slightly better results. That is logical, because most of the known aberrations (e.g., distortion) depend on the third power of radial distance.

may be identified with real stars and hence the situation cannot be remedied by restricting the list to objects identified with catalogued stars. Second, this approach allows one to estimate the limiting magnitude only within a local area and the quantity inferred does not characterise the entire image and is therefore unsuitable for quality control.

These problems can be addressed as follows. All stars of the reference catalogue that are located within the field considered are subdivided into magnitude bins of fixed width. For each bin the fraction of identified stars to the total number of catalogued stars is computed, and the corresponding histogram is drawn (see Figure 7). One can see that the ratio decreases sharply when the average magnitude of the bin approaches the limiting magnitude. In practice, it is convenient to adopt a certain threshold ratio and define the limiting magnitude as the magnitude at which the curve reaches this threshold. The thresholds of $\sigma_{\lim }=25 \%$ and $\sigma_{\lim }=50 \%$ have been shown empirically to correspond to objects with $S / N \approx 9-11 \sigma$ and $S / N \approx 14-15 \sigma$, respectively.

Note that for robust operation of the algorithm the limiting magnitude of the reference catalogue should be fainter than that of the frame studied. Otherwise the method becomes inapplicable because the catalogue lacks stars of the given magnitude. However, the USNO-B1 catalogue is sufficient for most of the modern wide-field surveys, whereas very wide-field surveys can be reduced successfully even with the TYCHO-2 catalogue (and this is very convenient).

\section{Automatic Classification and Analysis of Astronomical Transients}

3.1. Extraction of Objects and Data Transfer to the Server. The search for and classification of transients starts once 


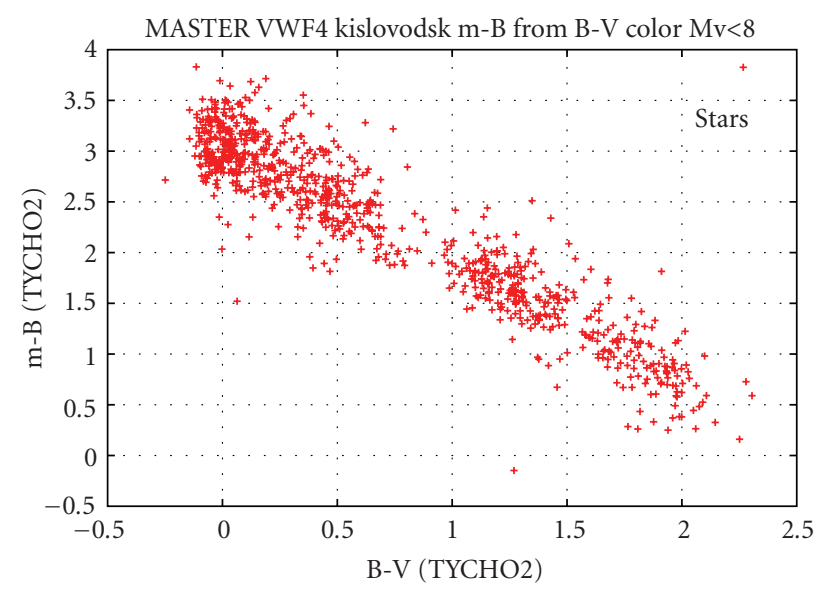

(a)

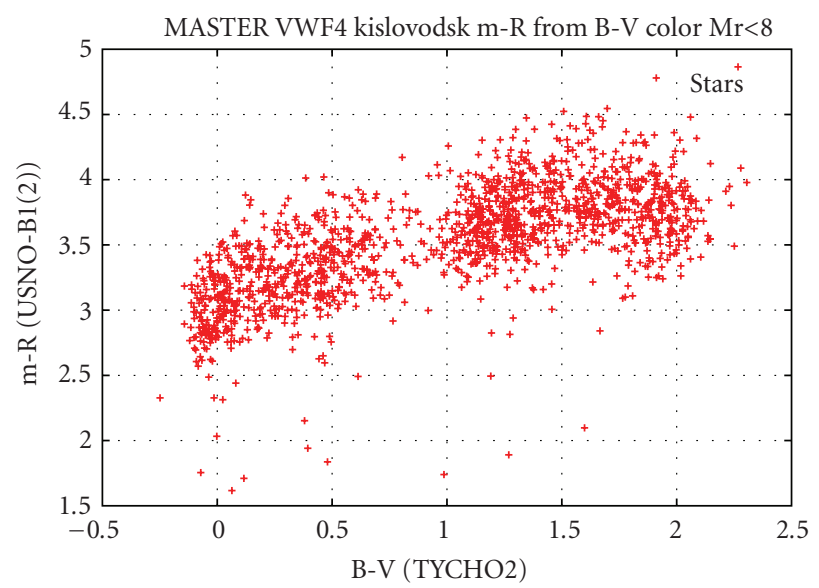

(c)

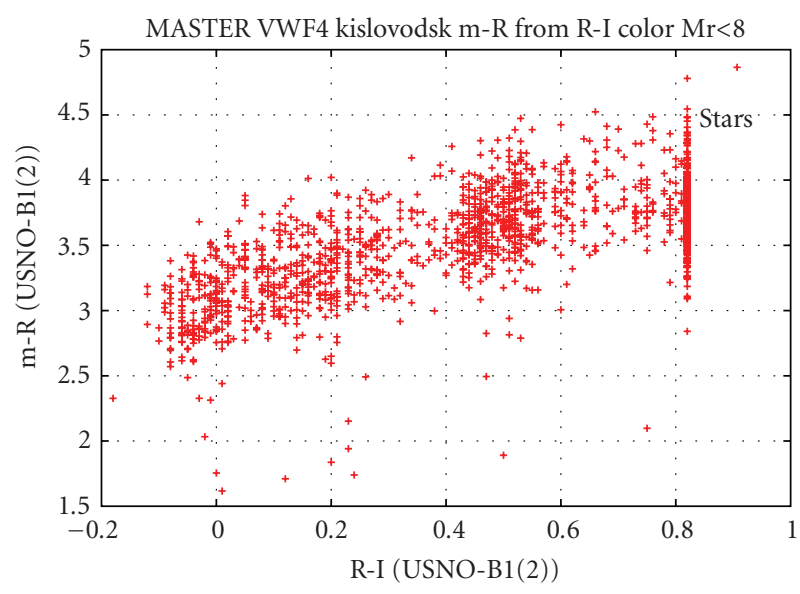

(b)

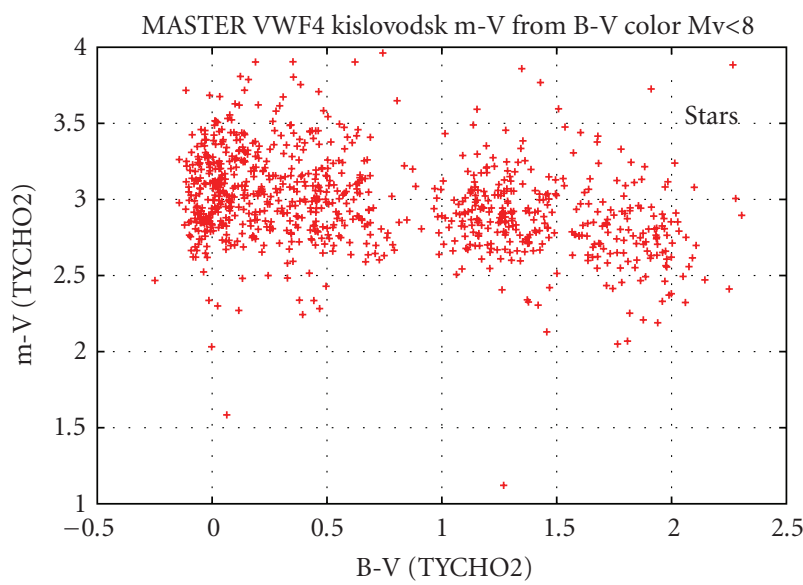

(d)

FIgURE 6: Color-color diagrams are used to calibrate instrumental magnitudes. On these plots $m=2.5 \lg \left(F_{\text {inst }}\right)$ and the zero point is of no importance and cannot be determined at the this stage. Note that the width of the sequences is real and due to the fact that stars have different spectral types, and not due to measurement errors. R-I $=0.82$ clustered connected with non very w.

the primary reduction of the image is finished. All transient events in frames can be subdivided into two classes: star-like objects as strips, ${ }^{3}$ which differ substantially in the methods used for primary reduction. All rapidly moving objects are usually classified as strips: low-altitude satellites and meteors. All transients of stellar or extragalactic nature (novas and supernovas, orphan bursts, stellar flares) and geostationary satellites (see, e.g., Figure 18) are Star-like. Star-like objects are much easier to process, because the program of object extraction and instrumental photometry (SExtractor, described above) has been developed to analyse such objects. Therefore all main parameters of Star-like objects (flux and magnitude, FWHM, Cartesian frame coordinates $(X, Y)$, and semiaxes $(a, b))$ are determined very reliably.

Extraction of strip-shaped objects is fraught with serious problems. First, a fine adjustment of SExtractor's additional internal parameters is necessary. Second, a change of these parameters strongly affects the image processing time. Furthermore, strip-shaped objects contribute appreciably to the background within the small area suitable for identification of stars. Fine adjustment of all parameters allowed us nevertheless not only to identify strips in the image, but also achieve high speed of operation (half exposure time) (see Figure 8). However, SExtractor algorithm cannot identify strips in the frame, to say nothing about performing their high-quality photometry. To this end, a special program had to be developed, which we describe below.

To reduce the server load, identification of objects in the image can be physically performed not only by the server, but also by the control computer of each particular camera (see Figure 3). Note that the resulting catalogue of detected objects should have higher priority for the transmission to the server compared to the images that are to be stored in the database.

3.2. Primary Reduction of Astronomical Transients. After the objects identification the next step is their astrometric and photometric calibration. A catalogue of objects with calibrated coordinates and photometry is produced. ${ }^{4}$ All unidentified objects (identification is performed using both coordinate and photometric criteria: usually $\Delta S=20-30^{\prime \prime}$ 


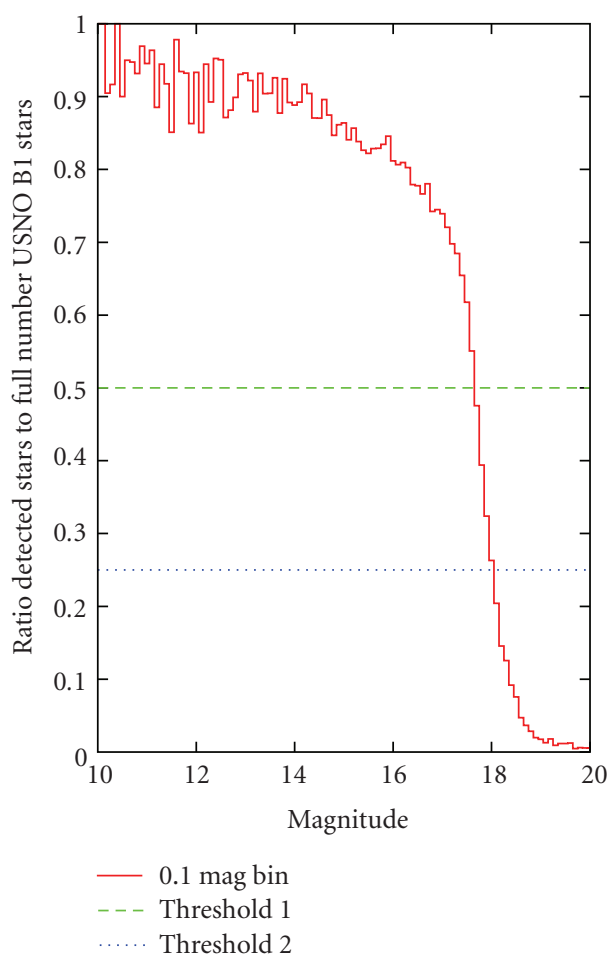

(a)

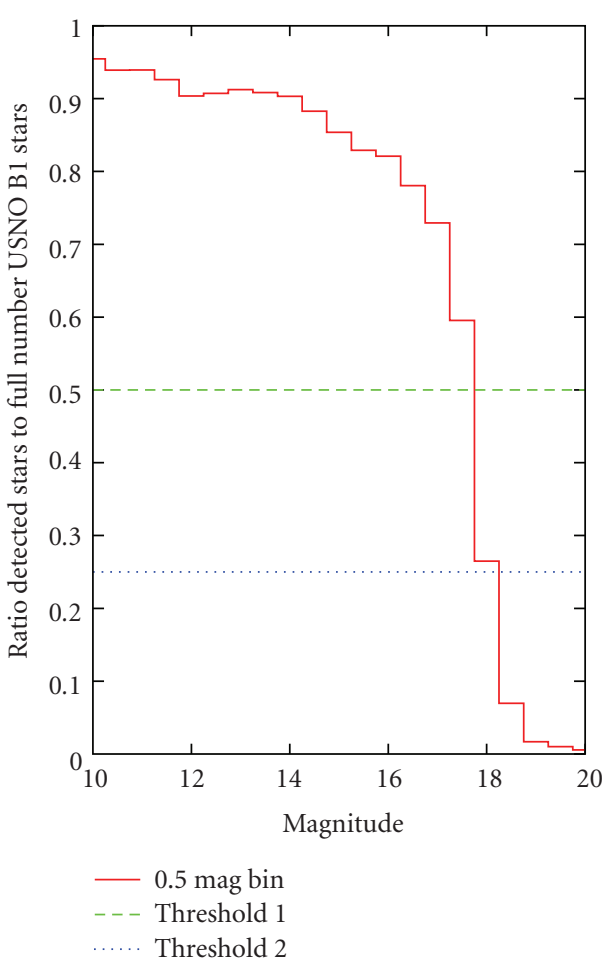

(b)

FIGURE 7: The number of identified stars as a function of magnitude. The straight lines indicate the $\sigma_{\lim }=50 \%$ and $\sigma_{\lim }=25 \%$ thresholds. Averaging was made within $0.5^{m}$ and $0.1^{m}$ bins in the right and left histograms, respectively. The right (coarser binned) histogram is smoother, resulting in reduced probability of accidentally reaching the threshold at a bright magnitude. It is therefore convenient to determine the limit on the coarsely binned histogram and then refine it on the finer-binned curve.

and $\Delta m=1.5^{m}$ ) are viewed as possible optical transients. This approach allows us to identify stellar flares as transients and to a certain extent prevent spurious identifications with fainter and hence more numerous stars. To filter noise, objects that could not be identified with the catalogue are correlated with the objects of several (usually 2-3) previous sets. An object is considered to be a candidate transient if it could not be found either in the catalogue or in the previous frames. All candidate transients are then passed through a number of special filters. For example, if the semiminor axis of the object, which should typically be of about $b \sim 0.7-2$ pixels, exceeds $b \sim 4-5$ pixels, it must be a trail of a cloud with a probability of $95 \% .{ }^{5}$ If FWHM $<1$ pixel, we must be dealing with a hot pixel or a cosmic-ray hit. We also set for all transients the trigger threshold, which is always higher than the extraction threshold. Currently, the trigger threshold is $S / N>10 \sigma$. We consider a candidate found on two or more consecutive frames to be a trustworthy transient.

Preliminary classification of transients is performed after the objects are passed through all filters. First, all objects are searched for possible identifications in the catalogue of artificial satellites and all identified objects are included into the database of satellites. The remaining ones are subdivided into Star-like and strip-shaped objects. The object is classified as a strip-shaped if $a / b>3$; otherwise it is considered to be Star-like (here $a$ and $b$ major and minor semiaxis). All Star-like objects are then passed through the coincidence circuit, and strip-shaped objects are. Objects are provisionally subdivided into meteor candidates and unknown artificial satellites. If each of three successive frames contains a candidate transient such that the three objects lie on a straight line, all of the them are considered to be the images of an artificial satellite. The remaining objects are considered to be meteor candidates.

3.3. Coincidence Scheme of and Determination of Heights. All the procedures described above are performed for each of the four cameras in real-time mode. Real-time processing ends here if the cameras operate in uncoupled (alert) mode. In the case of survey observations, when two cameras point to the same sky area, all Star-like transients are passed through the coincidence scheme, and all strip-shaped objects have their heights determined (see Figures 9 and 10). To improve the accuracy of identification, special transformation is applied to the frame taken with the Northern camera to convert it to the coordinate frame of the Southern camera. This transformation is based on the same principles as astrometric reduction. It takes little time to compute ( $\sim 0.2$ seconds) and improves the accuracy (and hence reduces the correlation radius and the number of chance identifications) by more than a factor of 2.5 compared to the accuracy of the results obtained in the case of identification by celestial coordinates. Should a real Star-like transient be found, an alert can be 


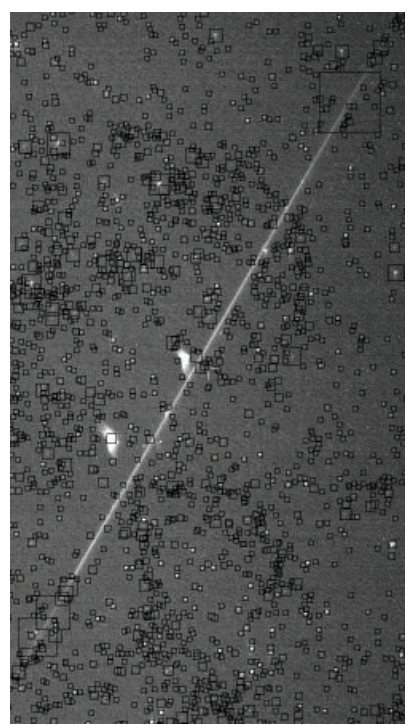

(a)

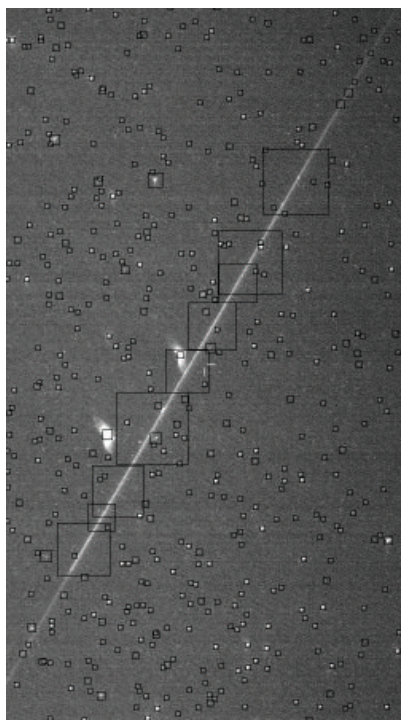

(b)

Figure 8: A $5^{\circ} \times 9^{\circ}$ image with a bolide. The black squares indicate objects extracted from the frame. The side of the square corresponds to the FWHM of the object. (a) shows a frame with objects extracted using standard SExtractor parameters (with the extraction threshold of $S / N>1.5$ ) with no filters applied. As a result, the program identified all Star-like objects, heaps of faint garbage, hot pixels, and simply random noise, that is, virtually everything except the bolide. (b) shows the result of extraction made using the currently adopted refined custom parameters. Several bright elongated objects are extracted on the bolide image and the noise level is appreciably lower.

immediately issued to larger $(45-\mathrm{cm})$ MASTER telescopes (located near Moscow, in Kislovodsk and in the Urals) to study the event in more detail. However, this mode is currently in the stage of development, mostly because of the debris of high-orbital satellites, which often contaminate the frames (see Figures 4 and 18).

3.4. Analysis of Strips. As we pointed out above, SExtractor computes only the approximate position of a strip, breaking it into several bright elongated objects. To perform complete photometry of a strip, determine the coordinates of its centre and ends and other parameters, a special program is used dedicated to the analysis of strips on astronomical images. To prevent server overload, this program is run only when cameras are idle (in the afternoon or during bad weather). The program starts with the coordinates of any location inside the strip determined at the previous stage. The working area of the given size (usually $512 \times 512$ pixels, which is sufficient for the analyses of any satellite or meteor trails) is chosen around this initial point. The size of the working area is automatically increased when a very bright and long bolide is to be analysed.

Determination of Background. To determine the background, we subdivide the working area into subareas of the given size. For each subarea the median and standard

deviation are computed twice: the first time over almost all pixels in the given subarea, and the second time more precisely, after discarding the pixels that deviate strongly (by more than $6-10 \sigma$ ) from the preliminary background estimate. We number all these subareas-there are a total of $N_{\text {zone }}$ of them (currently $N_{\text {zone }}=256$ ) - and compute the background and standard deviation at a given pixel as

$$
\begin{gathered}
\operatorname{Bg}(i, j)=\sum_{k=1}^{k<N_{\text {zone }}} \frac{1}{r_{k}^{2}} \cdot \operatorname{Med}(k), \\
\Sigma(i, j)=\sum_{k=1}^{k<N_{\text {zone }}} \frac{1}{r_{k}^{2}} \cdot \Sigma(k),
\end{gathered}
$$

where $\operatorname{Bg}(i, j)$ is the background at the point with coordinates $(i, j) ; \operatorname{Med}(k)$ is the median in $k$ th area; $\Sigma$ is the standard deviation within the corresponding subarea, $r_{k}$ is the distance between the point $(i, j)$ and the centre of $k$ th area. Such simple map suffices for the primary analysis. In a consequence more detailed analysis with more exact background definition (e.g., by SExtractor algorithm [8]) can be made. Figures 11: 3-4 show the corresponding background maps.

Extraction of Strips. To extract a strip, we first compute the map of bright pixels exceeding the background level by $n_{\sigma} \cdot \Sigma(i, j)$ or more. The optimum threshold has been shown empirically to be $n_{\sigma}=1.5$. The corresponding points are shown by black shade on the map (see Figure 11:2).

A recursive search algorithm for connected area is then used starting at the centre found by SExtractor. Once the connected area is found, the main parameters of the object (the position of the centre, $a, b$, and the angle $\theta$ between the axis of the object and the $X$ axis) are determined. The standard deviation $\sigma_{\text {line }}$ from the straight line drawn at angle $\theta$ through the centre of the object is computed if the object found can be classified as a strip $(a / b>3)$. The recursive algorithm of searching for connected area is then started again, this time within 2-3 $\sigma_{\text {line }}$ of the straight line just mentioned. The algorithm is allowed to jump over one to two faint pixels within $2 \sigma_{\text {line }}$ of the straight line. Thus the second pass yields a completely extracted strip including faint edges in the case of meteors.

The program then attempts to find objects located on the extension of the strip line on the subsequent and previous frames. If such object can be found, the strip is classified as a satellite; otherwise, as a meteor. The brightness profiles along the strip are then computed both for satellites and meteors (see Figure 12). Portions of images are kept for all transients.

\section{Synchronous Observations of Prompt GRB Emission}

In this section we discuss synchronous GRB observations. Note that during two years of the operation of the singlechannel wide-field Kislovodsk camera we have made over 20 faster than 1-minute alert pointings published in the corresponding GCN-telegrams GRB 070224 (gcn 6139), 


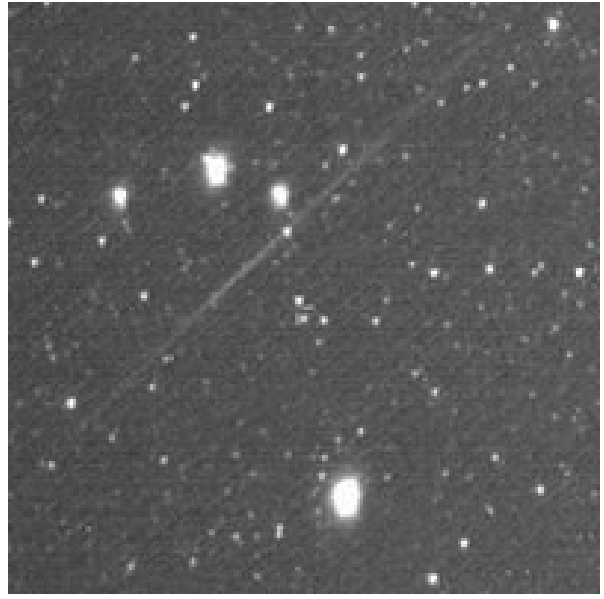

(a)

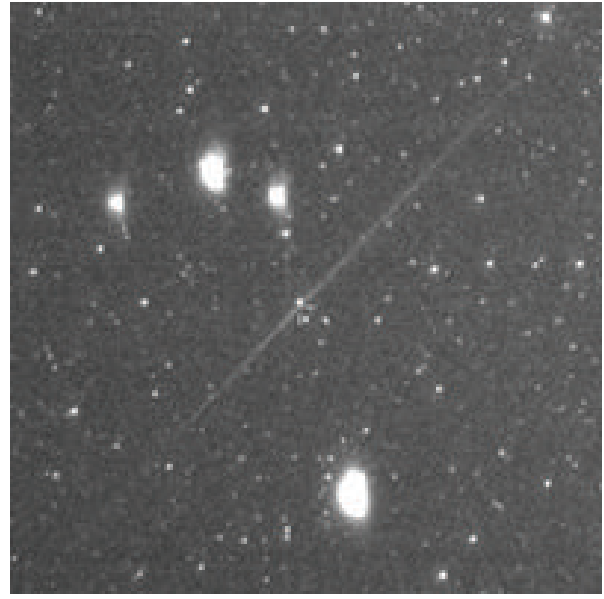

(b)

FIgURE 9: MASTER-VWF4 frames of a $5^{\circ} \times 5^{\circ}$ sky area taken with the Northern and Southern cameras. The parallax of the meteor is immediately apparent. Triangulation yields a height of $H_{\text {meteor }}=72 \pm$ of $2 \mathrm{~km}$.

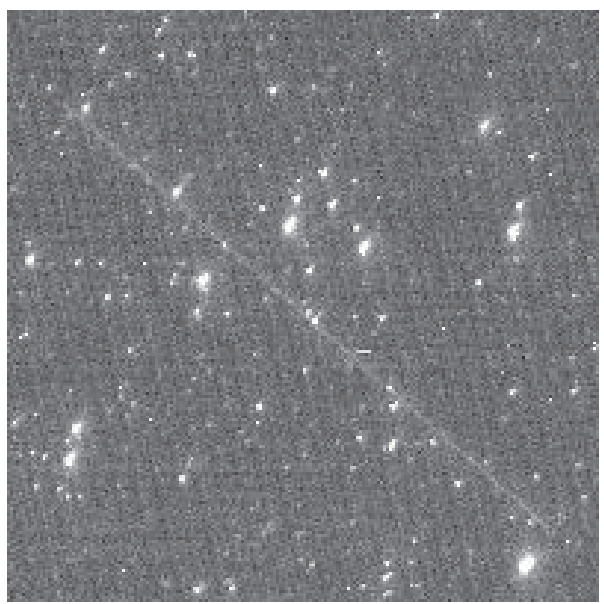

(a)

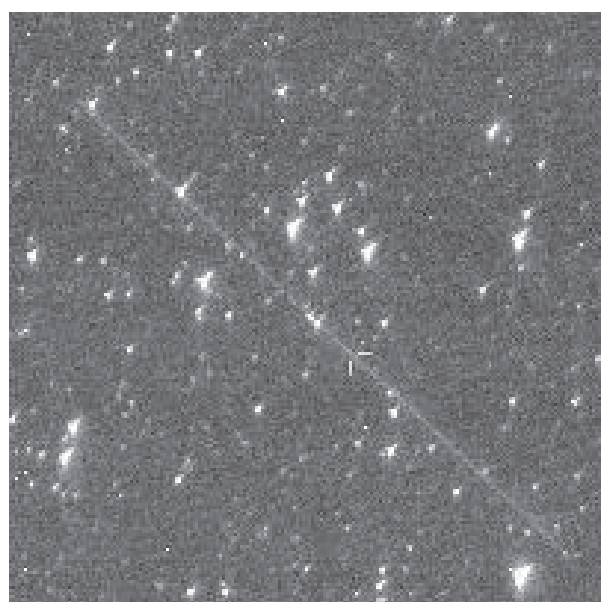

(b)

Figure 10: Parallax of an artificial satellite. Here we show the $4^{\circ} \times 4^{\circ}$ frames of a sky area taken with the MASTER-VWF4 Northern and Southern cameras. Triangulation yields a height of $H_{\text {satell }} \sim 4500 \mathrm{~km}$. Given the accuracy of astrometric measurements for strip-shaped objects, $\sigma_{\text {astrom }}^{\text {line }} \sim 20^{\prime \prime}$, and the baselength of $\Delta l=702 \mathrm{~m}$, the maximum height that can be determined via triangulation is $H_{\max }=\Delta l *$ $\arcsin \left(\sigma_{\text {astrom }}^{\text {line }}\right) \sim 10000 \mathrm{~m}$.

GRB 070223 (gcn 6131), GRB 070219 (gcn 6113), GRB 061213 (gcn 5915), GRB 061002 (gcn 5677), GRB 060929 (gcn 5657), and others [9].

4.1. The Importance of Synchronous Observations. As is well known, optical GRB glow can be roughly subdivided into two parts: prompt emission and afterglow. We do not discuss the afterglow phenomenon here, because it has already been thoroughly studied and it is not the target of Wide-Field observations. Let us now consider prompt emission.

Prompt optical GRB emission is the emission that appears simultaneously with gamma rays detected by various space-based gamma observatories (Hete2, Swift, Fermi). Unlike afterglow, it bears information about the object that gives birth to GRB, and not about its environment. There are two ways to detect prompt optical GRB emission: synchronous observations and very quick alert pointing. To date, about a dozen [10] optical prompt GRB have been made and most of them were alert based. That is to say, when prompted by a quick trigger from a gammaray observatory, a robotic optical telescope points at the object within several tens of seconds after the corresponding gamma-ray event is detected. If the GRB is long enough, its prompt emission is still detectable by this time. Another, less common type of observations, are synchronous wide-field camera observations. Perhaps the most amazing example of the latter is the prompt GRB 080319B detection [1]. Synchronous observations are much more efficient than alert observations for at least two reasons. First, alert observations 


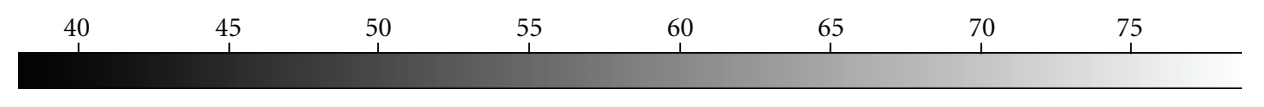
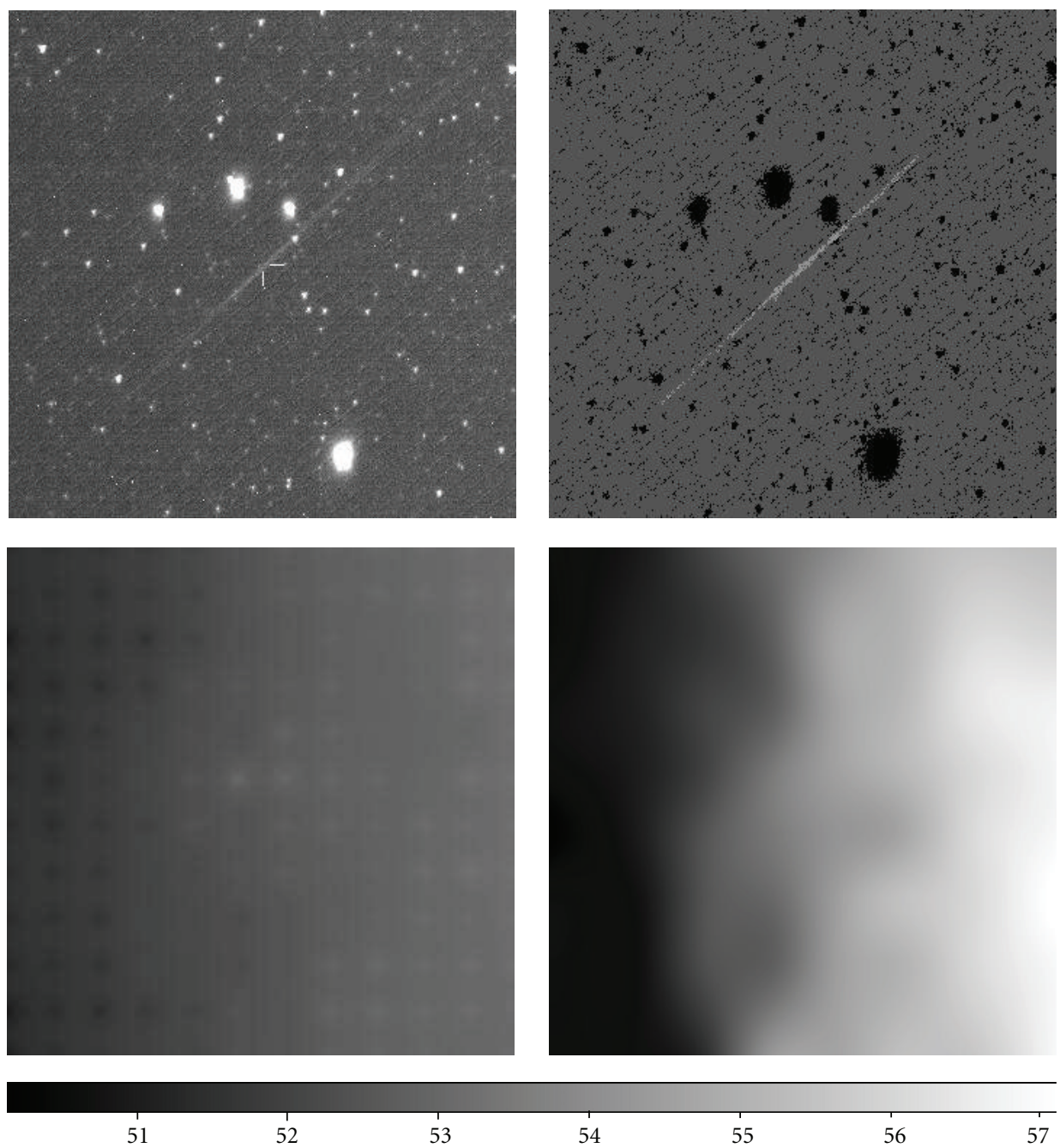

FIGURE 11: (1) Image of a meteor on a $3^{\circ} \times 3^{\circ}$ frame $(F(i, j))$. (2) Extraction map. Black shade shows the points with $F(i, j)>n_{\sigma} \cdot \Sigma(i, j)$; white shade indicates the points included into the object under study. Grey shade indicates the background. (3) Background map- $(\operatorname{Bg}(i, j))$ (bottom left). (4) More exact map of a background use SExtractor background estimation algorithm [8].

are subject to selection effects. The point is that an alert may result in the detection of prompt emission only if the GRB lasts longer than 30-40 seconds, because of the delay due to the time it takes to perform data processing on-board the space telescope, transmit the signal to the ground, and point the optical telescope. Hence alerts cannot be used to detect prompt optical emission from short GRBs. It is significant that this kind of emission has never been observed and moreover, it has never had its upper limit measured.

\subsection{Energy Calibrations of the MASTER-VWF4 Photometric} Passband. To make any conclusions about the brightness of objects not just in terms of relative magnitudes, but also in terms of energy units, we must calibrate the MASTERVWF4 zero-point. Let us determine the energy flux (in the units if $\mathrm{erg} / \mathrm{cm}^{2} / \mathrm{s}$ ) produced by a zero-magnitude star (e.g., Vega). The MASTER-VWF4 band is close to the $V$ band of the Tycho2 catalogue. Figure 13 shows the spectrum of Vega and the same spectrum folded with the lens passband and sensitivity curve of the CCD. Integration over the entire spectrum finally yields the flux produced within the MASTER-VW4 band by a zero-magnitude star:

$$
F^{\mathrm{Vega}}=\int_{0}^{\infty} f_{v}(\nu) \cdot \nu \cdot d \nu=6.4 \pm 0.1 \cdot 10^{-6} \mathrm{erg} / \mathrm{cm}^{2} / \mathrm{s}
$$

4.3. Synchronous GRB Observations with MASTER-VWF Very Wide-Field Cameras. MASTER-VWF cameras perform nightly monitoring of the sky with the aim to detect optical transients. These cameras may operate in two different modes. In the first mode, which is meant for searching for optical transients, two cameras are pointed to the same 


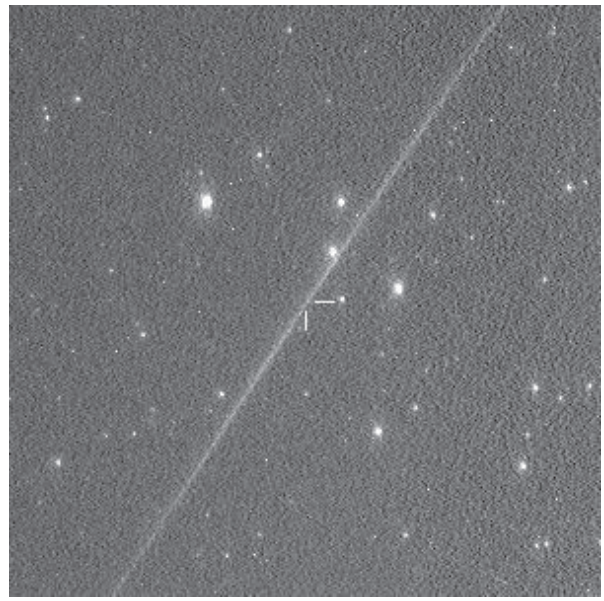

(a)

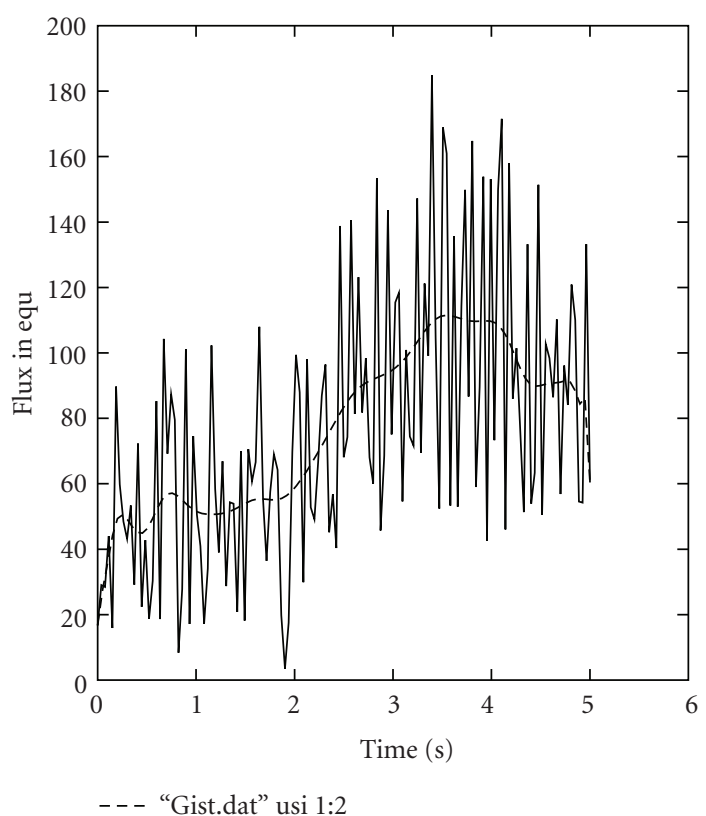

(b)

FIGURE 12: Meteor and its automatically determined brightness profile, FOV is about $5^{\circ} \times 5^{\circ}$ here.

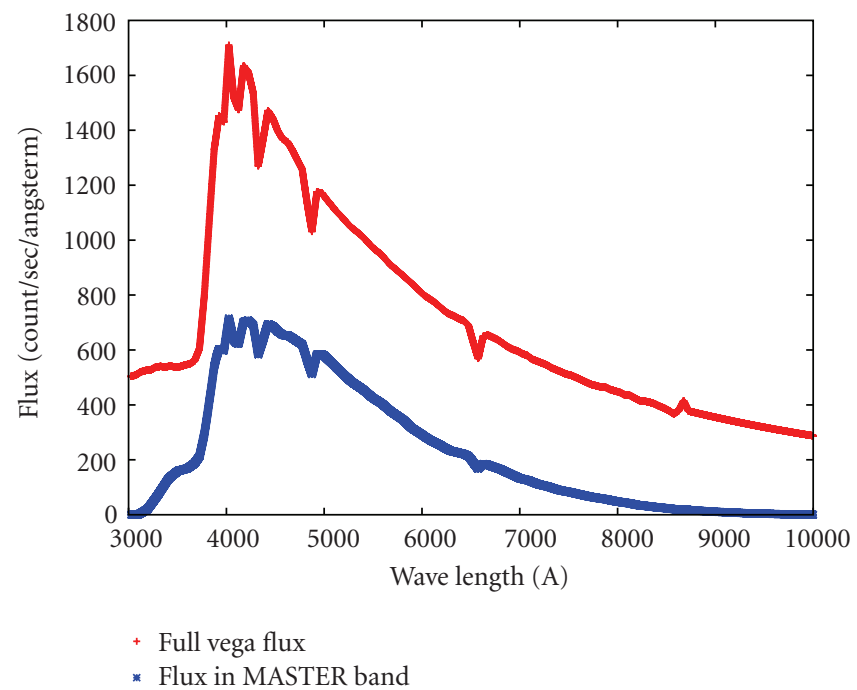

(a)

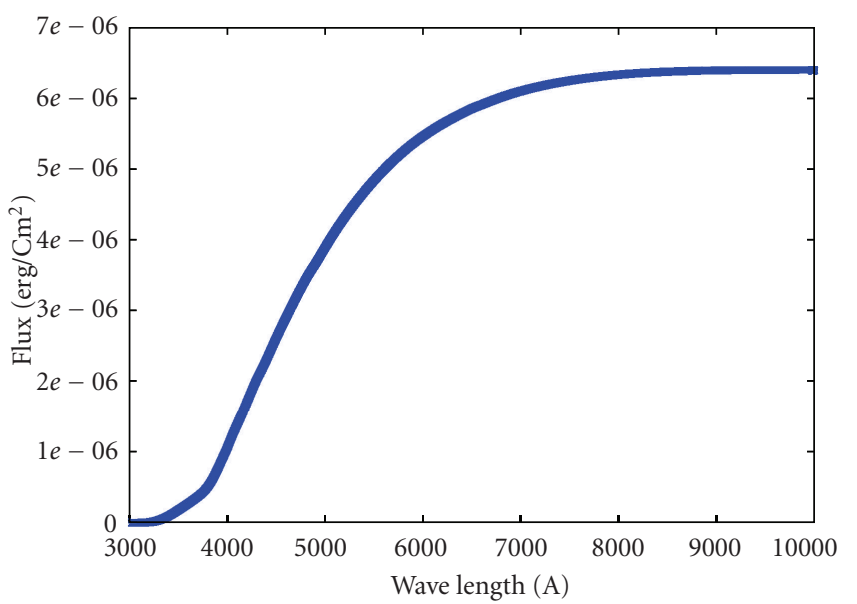

* Flux in MASTER band

(b)

FIGURE 13: The spectrum of Vega and the same spectrum as observed after passing through the MASTER-VWF band (a). The cumulative curve of this spectrum in power units (b).

sky area in order to implement the coincidence scheme (see Figures 9 and 10). In the second mode the fields of view of the cameras are set apart as far as possible in order to provide a combined field of view of more than 4000 and $2000 \mathrm{deg}^{2}$ for the Kislovodsk and Irkutsk observatories, respectively. The second mode is dedicated for synchronous observations of gamma-ray bursts. gamma-ray observatory satellite have very accurate coordinates, allowing the location of the burst origin on very widefield cameras to be determined with the highest precision possible (i.e., to within less than one-pixel large error box).

Long Gamma-Ray Burst GRB081102. (see Figure 14) [15] Although it was a typical long $\left(T_{90}=63 \mathrm{~s}\right)$ gamma-ray burst, the telegram from Swift was issued only after 15 minutes. This delay prevented early observations by automated telescopes. However, MASTER-VWF-4 in Kislovodsk covered 


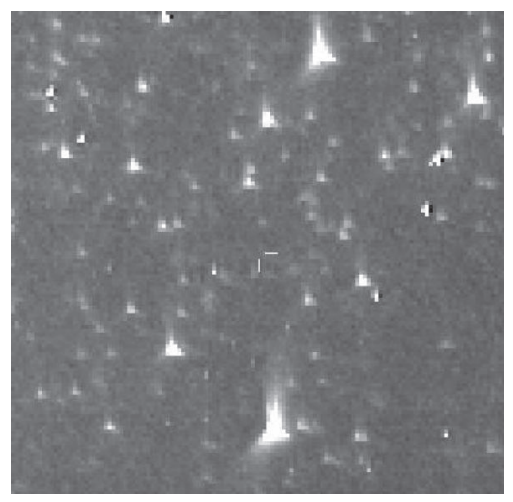

(a)

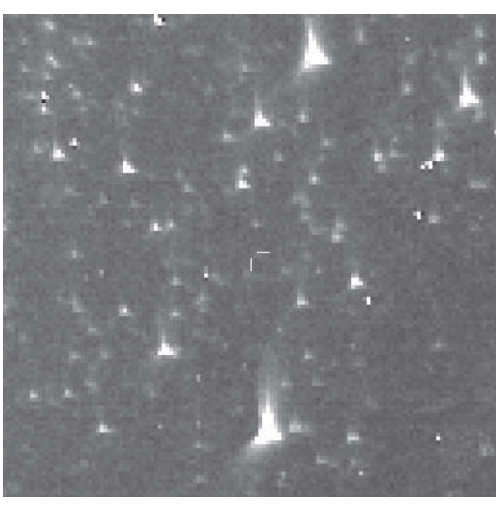

(b)

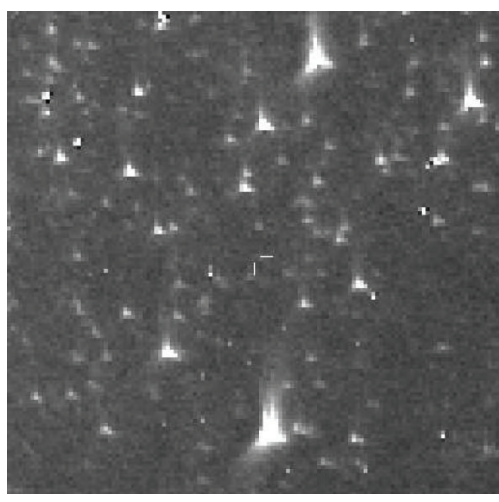

(c)

FIGURE 14: Coadded images taken 30 seconds before (a), during (b), and 30 seconds after (c) GRB081102. Each frame is a coadd of 12 images (six images from each camera) -60 seconds exposure for each frame. FOV $3^{\circ} \times 3^{\circ}$ on the frame edge. So strange stars shape is connected with off-axis aberrations. Animation is available here: http://observ.pereplet.ru/images/GRB081102/grb_film.html.

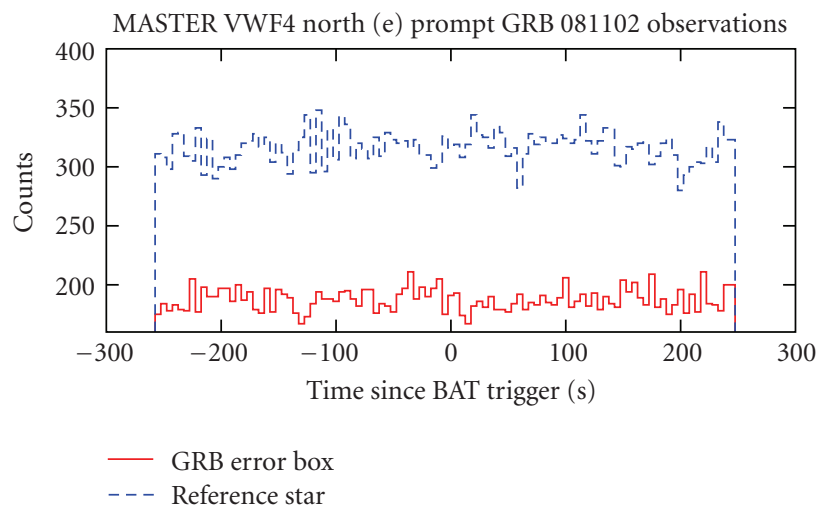

(a)

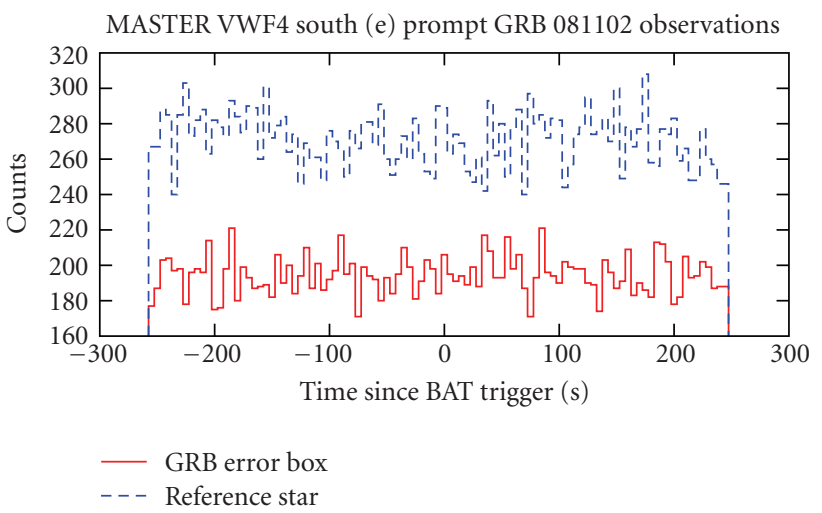

(b)

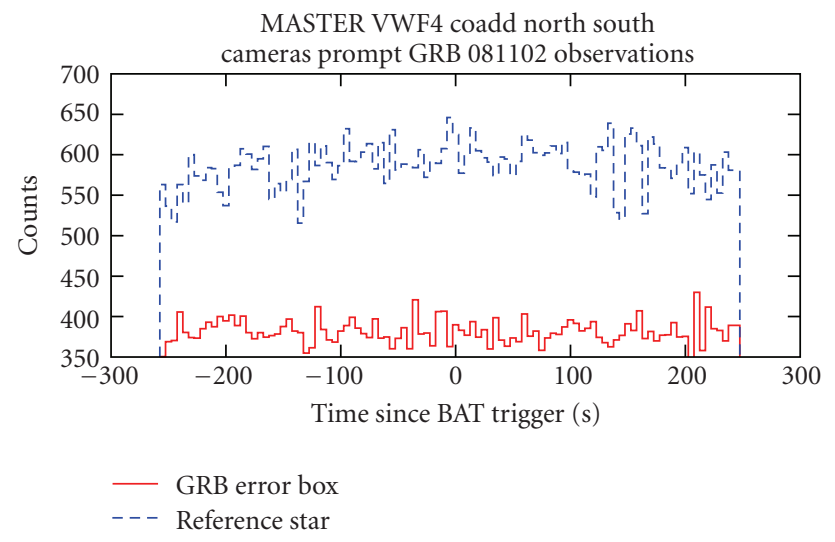

(c)

Figure 15: GRB081102. Hash (red) from the Northern and Southern cameras and from the two cameras simultaneously inside the GRB081102 error-box and the signal from the $V_{\text {ref }}=11.5^{m}$ reference star (blue).

the entire error box at the edge of its field of view. MASTERVWF-4 operated in the survey mode with aligned cameras, allowing the grb error-box to be recorded simultaneously in two channels. This area has been observed for two hours before and seven hours after the grb with no time gaps [16].
Unfortunately, no optical counterpart was detected from this burst (see Figures 14 and 15). The lack of optical counterpart may have been due to extremely strong absorption toward the burst (formula 4). However, a very high upper limit (for synchronous (!) observations with very wide-field 


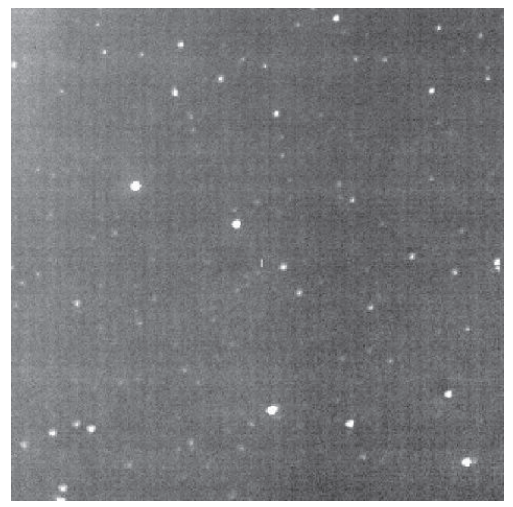

(a)

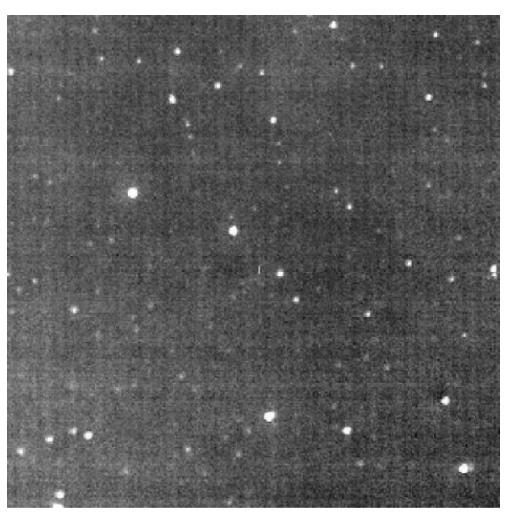

(b)

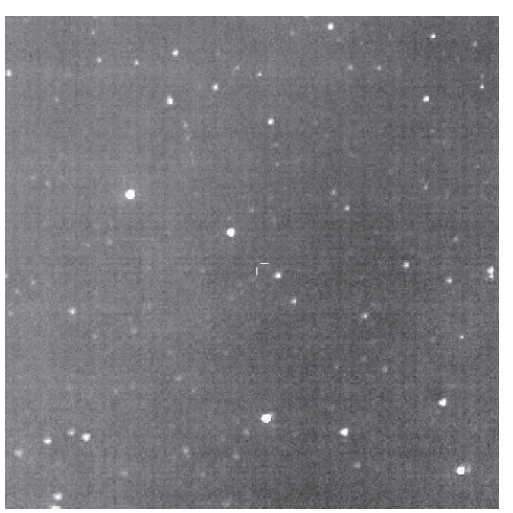

(c)

FIGURE 16: A $6^{\circ} \times 6^{\circ}$ field around the GRB090424 Swift XRT position. One-minute exposure images taken before, during, and after $T_{\mathrm{GRB}}$. Clouds can be seen on the images, resulting in the optical limit of $V<9.7^{m}$ on each frame. Animation is available here: http://observ.pereplet.ru/images/film_grb.html.

cameras) of $V_{\text {grb081102 }}<13.0^{m}$ has been obtained, which is the highest synchronous upper limit so far [17].

Let us now compare GRB081102 with GRB080319B from which bright prompt optical emission $V=5.3^{m}$ [1] has been recorded. Consider the $F_{\text {opt }} / F_{\gamma}$ ratio (where $F_{\text {opt }}$ and $F_{\gamma}$ are the optical and gamma-ray flux, resp.) for both bursts. The gamma-ray spectrum is known for all bursts discussed in this paper and therefore we reduce $F_{\gamma}$ to the flux in the 15-150 keV energy interval for the sake of uniformity.

Let us estimate $F_{\mathrm{opt}}^{\mathrm{grb} 01102}$. The $V$-band extinction can be estimated by the following simple empirical formula [18]:

$$
\tau_{V}=5.2 \cdot 10^{-22} N_{H} .
$$

Given the known hydrogen column density toward GRB081102, $N_{H}=4.9 * 10^{21} \mathrm{~cm}^{-2}$ [19], we can use the inferred optical depth and Pogson's formula to estimate $F_{\gamma}^{\mathrm{grb} 081102}=2.3 \cdot 10^{-6} \mathrm{erg} / \mathrm{cm}^{2}[20]$ or

$$
\begin{gathered}
\tau_{V}^{\mathrm{grb} 081102}=5.2 \cdot 10^{-22} \cdot 4.9 \cdot 10^{21}=2.548=>\delta m=2.8^{m}, \\
\frac{F_{\mathrm{opt}}^{\mathrm{grb} 081102}}{F_{\gamma}^{\mathrm{grb} 081102}}<\frac{2.512^{m^{\mathrm{Vega}}-\left(m^{\mathrm{grb} 081102}-\delta m\right)} \cdot F^{\mathrm{Vega}} \cdot T_{90}^{\mathrm{grb} 081102}}{F_{\gamma}^{\mathrm{grb} 081102}}, \\
\frac{F_{\mathrm{opt}}^{\mathrm{grb} 081102}}{F_{\gamma}^{\mathrm{grb} 081102}}<\frac{2.512^{0-13.0+2.8} \cdot 6.4 \cdot 10^{-6} \mathrm{erg} / \mathrm{s} / \mathrm{cm}^{2} \cdot 63 \mathrm{~s}}{2.3 \cdot 10^{-6} \mathrm{erg} / \mathrm{cm}^{2}}=\frac{1}{83} .
\end{gathered}
$$

The "less than" sign is used because only the upper limit could have been determined for grb081102. For GRB080319B the column density is $N_{H}=9.2 * 10^{20} \mathrm{~cm}^{-2}$ [1], that is, five times lower than toward GRB081102 $\tau_{V}^{\text {grb080319B }}=0.48, F_{\gamma}=8.25 \cdot 10^{-5}=>\delta m=0.5^{m}$. Hence

$$
\frac{F_{\mathrm{opt}}^{\mathrm{grb} 080319 \mathrm{~B}}}{F_{\gamma}^{\mathrm{grb} 080319 \mathrm{~B}}}=\frac{1}{22} \text {. }
$$

It thus follows that the fraction of prompt optical emission for GRB081102 is at least four times smaller than for grb080319B.
The Long Gamma-Ray Burst GRB090424. At that time MASTER-VWF-2 in Irkutsk operated in the alert mode (with cameras pointed apart) and GRB090424 happened to be at the centre of the field of view of one of the cameras (Figure 16). It was also a typical long $\left(T_{90}=48 \mathrm{~s}\right)$ gammaray burst and UVOT detected the afterglow with unfiltered magnitude of about $15.3^{m} 167$ seconds after the burst [21]. MASTER-VWF-2 observed the grb error-box without time gaps for 1.2 hours before, during and 1.5 hours after the gamma-ray burst with 1-second exposures. Unfortunately, weather conditions were far from favourable, resulting in a not so high upper limit of $V_{1 s}<8.0^{m}$ and $V_{60 s}<60.0^{m}$ for a coadd of 60 images (see [22] and [23]). According to both Swift/BAT [24] data and FERMI observatory data converted to the $15-150 \mathrm{keV}$ energy interval [25], $F_{\gamma}^{\text {grb090424 }}=2.1 \cdot 10^{-5}$, implying that

$$
\frac{F_{\mathrm{opt}}^{\mathrm{grb} 090424}}{F_{\gamma}^{\mathrm{grb} 090424}}<\frac{1}{530} .
$$

Note that despite the rather modest upper limit the burst was very powerful and even such an upper limit restricts substantially the possible flux.

4.3.2. Gamma-Ray Bursts Recorded by FERMI Observatory. Unlike Swift, the FERMI observatory does not specialise in gamma-ray burst observations. Its on-board equipment allows the burst coordinates to be determined only to within several degrees or even worse. FERMI currently issues two to three times more alerts than Swift and is capable of recording harder (up to $10 \mathrm{MeV}$ ) radiation (Swift has an upper sensitivity limit of about $300 \mathrm{keV}$ ), and responds to short hard gamma-ray bursts, whose prompt optical or IR emission has not been detected so far. Given the large error of the coordinate measurement, gamma-ray bursts detected by FERMI observatory may at present (and, very likely, also in the future) have their prompt emission observed only with wide-field cameras, such as MASTER-VWF. 


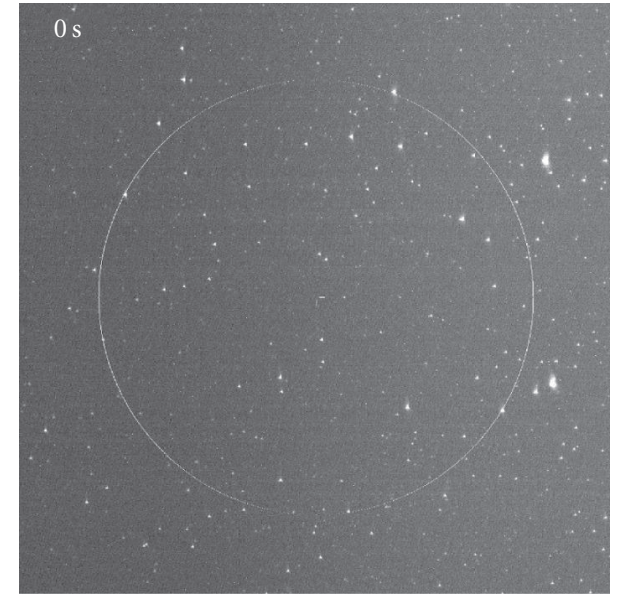

FIgURe 17: The most recent error box of GRB081130B $\alpha=$ $00^{h} 56^{m} 20^{s} \delta=+04^{\circ} 12^{\prime}$, error-box radius is $R=3.5^{\circ}$ [11]. The centre of the field of view of the camera that which recorded the burst pointed at $\alpha=01^{h} 01^{m} 56^{s} \delta=+19^{\circ} 20^{\prime} 38^{\prime \prime}$. The image size is $10^{\circ} \times$ $10^{\circ}$. Animation available here: http://observ.pereplet.ru/images/ GRB081130B/grb_film.html.

Until now (July, 1 2009), a total of five FERMI GRB error boxes have overlapped with the MASTER-VWF field of view, and two more (GRB081215 and GRB090526) proved to be outside the field of view after a very substantial final refinement of their coordinates.

GRB081130. GRB081130 was the first burst recorded by FERMI and synchronously observed by the MASTER-VWF4 facility in Kislovodsk. Its coordinates and error-box have been refined repeatedly (with a characteristic scatter of $\pm 10^{\circ}$ ), but always fell within the field of view of our cameras. Figure 17 shows the final localisation of this gamma-ray burst. The error box of grb081130 has been observed without time gaps for several hours before, during, and for hours after the grb.

No proper candidates could have been found inside the error box. However, given the rather large uncertainty of the coordinates measured by FERMI, we extended the search area for transient candidates to the entire frame. As a result, a very interesting object resembling a grb optical counterpart has been found 14 degrees from the localisation centre synchronously with grb (Figure 18). However, after cross identification with the catalogue of artificial satellites this transient was found to coincide with a high-orbit satellite, which occasionally produces such flashes. Thus a rather high upper limit of $V_{\text {grb081130B }}>12^{m} .0$ has been obtained again after the coadd of four images $\left(T_{\text {grb081130B }}=15-20 \mathrm{~s}\right.$ taken in different channels [11]) [26].

When converted to the $15-150 \mathrm{keV}$ energy interval, the gamma-ray fluence from GRB081130 is $F_{\gamma}^{\text {grb081130B }}=1.01$. $10^{-6} \mathrm{erg} / \mathrm{cm}^{2}$. We then apply the same procedures as in the case of GRB081102 to infer $F_{\mathrm{opt}}^{\mathrm{grb}}=3.82 \cdot 10^{-9} \mathrm{erg} / \mathrm{cm}^{2}$, implying a flux ratio of $F_{\mathrm{opt}}^{\mathrm{grb} 081130 \mathrm{~B}} / F_{\gamma}^{\mathrm{grb} 081130 \mathrm{~B}}<1 / 245$ [27].
GRB090305B. The gamma-ray burst GRB090305 (Figure 19) was recorded by FERMI [12]. MASTER-VWF-4 in Kislovodsk operated in the alert mode (with cameras apart) and observed $80 \%$ of the GRB090305 error-box at the edge of the field of view of one of the cameras. Despite of its rather short duration $\left(T_{90}=2 \mathrm{~s}\right)$ [12], GRB090305 was nevertheless a rather long and bright gamma-ray burst. However, even the final position estimate has a large errorbox $\left(\alpha=10^{h} 20^{m}, \delta=68^{\circ} 06^{\prime} R_{1 \text { sigma }}=5.4^{\circ}\right)$ and therefore no alert observations have been made. MASTER-VWF-4 has observed the error-box of this GRB without time gaps for 3.5 hours before, during and for two hours after the with 1-second exposures. No optical transients close to $T_{\mathrm{GRB}}$ could be found and we obtain an upper-limit of $V_{1 s}<9.5^{m}$ [28]. $F_{\mathrm{opt}}^{\mathrm{grb} 000305 \mathrm{~B}} / F_{\gamma}^{\mathrm{grb} 090305 \mathrm{~B}}<1 / 121$.

GRB090320B. The MASTER-VWF-4 system in Kislovodsk observed synchronously $80 \%$ of the GRB090320B errorbox (see Figure 20). This moderately bright burst has been detected by FERMI observatory at $\alpha=12^{h} 15^{m} .3, \delta=57^{d} 33^{\prime}$ with an uncertainty of 12.6 degrees (this includes the $3^{\circ}$ systematic error) [13]. No optical candidates could be found for this burst, which was long enough $\left(T_{90}=60 \mathrm{~s}\right)$ for its upper-limit $V<11^{m}$ to be estimated on 60 -s. exposure sets [29].

On the whole, this burst turned out to be rather faint with $F_{\mathrm{opt}}^{\mathrm{grb} 090320 \mathrm{~B}} / F_{\gamma}^{\mathrm{grb} 090320 \mathrm{~B}}<1 / 57$.

GRB090328B. Finally, GRB090328B is the last of the bursts considered here to deserve a separate discussion. It had 20\% of its error box observed by MASTER-VWF-2 in Irkutsk (Figure 21). GRB090328B was short compared to all other long bursts and it is the first short gamma-ray burst to have been synchronously observed.

This faint burst has been recorded FERMI observatory at 19:13:46.1 UT on 20 March 2009 at $\alpha=10^{h} 22^{m} .8, \delta=$ $33 \mathrm{~d} 24^{\prime}$ with with an uncertainty of $26.82^{\circ}$ (including the $3^{\circ}$ systematic error) $[26,30]$. It is the faintest burst so far recorded by our cameras and had therefore its coordinates determined with large errors. We found its upper limit to be $V<9.1^{m}$ on $1 \mathrm{~s}$ images and $F_{\mathrm{opt}}^{\mathrm{grb} 090328 \mathrm{~B}} / F_{\gamma}^{\mathrm{grb} 090328 \mathrm{~B}}<1 / 1527$ [14].

\section{Summary}

Figure 1 and Table 2 summarise the results of observations of prompt emission of gamma-ray bursts. Even a cursory analysis of Figure 1 demonstrates that optical emission is better correlated with soft rather than hard gamma-ray emission. The gamma-ray burst GRB090424 is located in a remarkable position in Figure 1. One can see that the upper limit for its optical emission is about one order of magnitude lower than the flux from the famous gammaray burst GRB990123, a pattern that may be indicative of irregular conversion of gamma-ray emission into optical radiation from one burst to another. On the whole, it is safe to conclude that more observations are required for a detailed analysis. The example of GRB090424 gamma-ray 


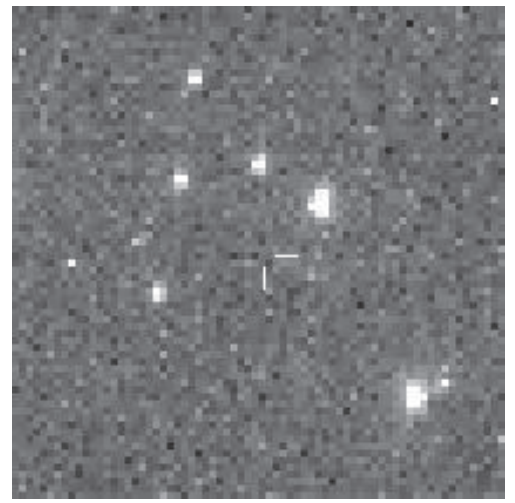

(a)

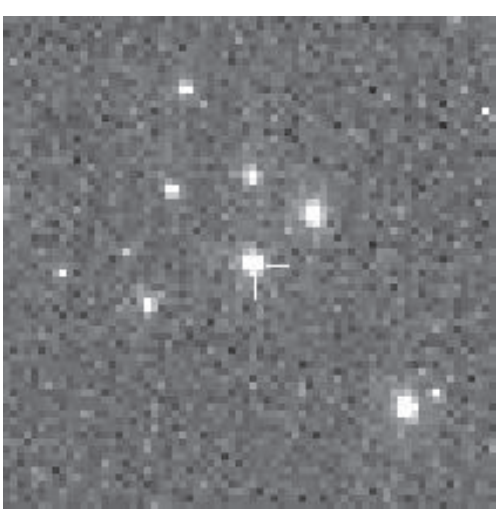

(b)

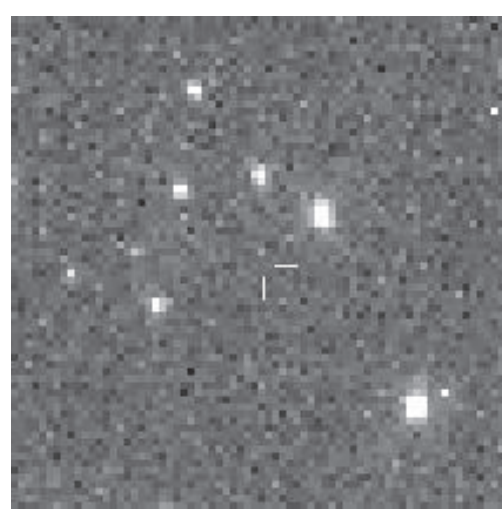

(c)

FIGURE 18: Transient object found simultaneously with GRB081130B at $\alpha=01^{h} 10^{m} 20^{s} \delta=+18^{\circ} 44^{\prime} 35^{\prime \prime}$, 14 degrees from the centre of its last GRB081130B localisation. A detailed analysis showed the object to be a short flash produced by the "MOLNIYA" high-orbit satellite.

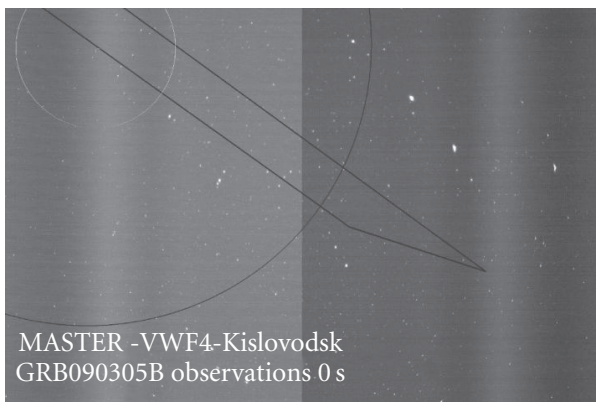

Figure 19: MASTER-VWF4 Kislovodsk GRB090305B follow-up observation. This is a $40^{\circ} \times 24^{\circ}$ full-frame image. The white and black circles show the error boxes corresponding to the 1 and 3 sigma FERMI error limits plus the $3^{\circ}$ systematic error [12] $\left(R_{\text {lsigma }}=5.4^{\circ}\right)$. The black rectangle shows the IPN triangulation error-box Animation is available here http://observ.pereplet.ru/ images/GRB090305B/GRB090305B_full2.gif.

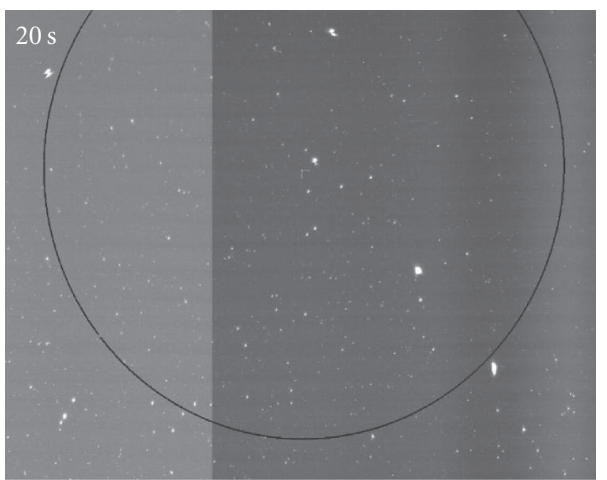

Figure 20: MASTER-VWF4 Kislovodsk GRB090320B follow-up observation. This is a $40^{\circ} \times 24^{\circ}$ full-frame image. The black circle shows the error box corresponding to the 3 sigma FERMI error limits plus the $3^{\circ}$ systematic error $\left(R=12.6^{\circ}\right)$ [13]. Animation is available here http://observ.pereplet.ru/images/GRB090320B/ GRB090320B_60sec.gif.

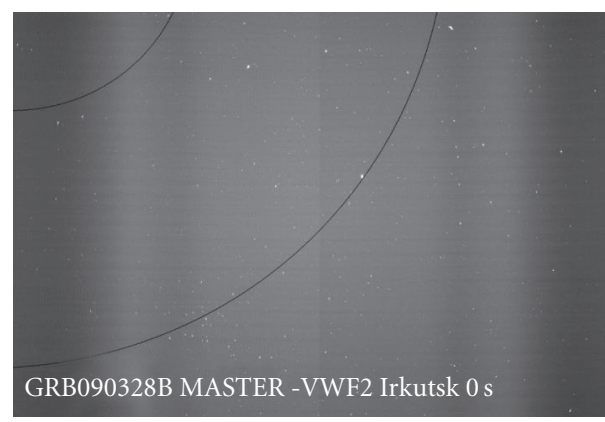

Figure 21: MASTER-VWF4 follow-up observations of GRB090328B in Kislovodsk. This is a $40^{\circ} \times 24^{\circ}$ full-frame image. The black circle shows the error box corresponding to the 3 sigma FERMI error limits plus the $3^{\circ}$ systematic error $\left(R=12.6^{\circ}\right)$ [14]. Animation is available here http://observ.pereplet.ru/images/ GRB090320B/GRB090320B_60sec.gif.

burst indicates that even the measured optical limit may constitute a result of real importance and that and this burst deserves separate analysis.

In this article we describe the methods of observation and analysis of images taken with very wide-field cameras, and their application to synchronous observations of prompt gamma-ray burst emission in the MASTER-VWF experiment. We finally recall that the renowned astrophysicist Bohdan Paczynski [31] pointed out in his last paper the exceptional astrophysical significance of robotic telescope networks, which, like MASTER, are composed of Wide-Field searching cameras and larger telescopes. Now his and our ideas at last become reality.

\section{Acknowledgment}

This work was supported by the Ministry of Science of the Russian Federation (state contract no. 02.740.11.0249). 


\section{Endnotes}

1. The information about archive opening will be accessible on our website and will published separately.

2. Original program is delivered by E. Gorbovskoy and A. Belinskii.

3. As Star-like I name objects with $1.5<$ FWHM $<4-5$ and the small semiaxes ratio $a / b<2-3$. Limiting the relation of semiaxes it is so great because objects at frame edges have naturaly prolated the form (see stars form on Figure 8 or 14).

4. For pipeline processing we use only dark and bias calibrations. Long and more computationally difficult flat-calibration is used only necessarily (GRB case for an example).

5. Semimajor axis (a) unused here because if can change in wast limits for real objects. From 1.5-3 for stars to 100 for fast satellites.

\section{References}

[1] J. L. Racusin, S. V. Karpov, M. Sokolowski, et al., "Broadband observations of the naked-eye $\gamma$-ray burst GRB 080319B," Nature, vol. 455, no. 7210, pp. 183-188, 2008.

[2] M. Ćwiok, W. Dominik, K. Małek, et al., "Search for GRB related prompt optical emission and other fast varying objects with "Pi of the Sky" detector," Astrophysics and Space Science, vol. 309, no. 1-4, pp. 531-535, 2007.

[3] V. M. Lipunov, V. G. Kornilov, A. V. Krylov, et al., "Optical observations of gamma-ray bursts, the discovery of supernovae 2005bv, 2005ee, and 2006ak, and searches for transients using the "MASTER" robotic telescope," Astronomy Reports, vol. 51, no. 12, pp. 1004-1025, 2007.

[4] K. Onda, T. Tamagawa, M. Tashiro, et al., "Ultra wide-field telescope WIDGET for observing GRB," Nuovo Cimento della Societa Italiana di Fisica B, vol. 121, no. 12, pp. 1549-1550, 2006.

[5] S. Karpov, G. Beskin, A. Biryukov, et al., "Optical camera with high temporal resolution to search for transients in the wide field," Nuovo Cimento della Societa Italiana di Fisica C, vol. 28, no. 4-5, pp. 747-750, 2005.

[6] E. Molinari, S. Bondar, S. Karpov, et al., "TORTOREM: twotelescope complex for detection and investigation of optical transients," Nuovo Cimento della Societa Italiana di Fisica B, vol. 121, no. 12, pp. 1525-1526, 2006.

[7] V. Lipunov, V. Kornilov, E. Gorbovskoy, et al., "Master robotic net," Advances in Astronomy, vol. 2010, Article ID 349171, 6 pages, 2010.

[8] E. Bertinl, "SExtractor: software for source extraction," Astronomy and Astrophysics Supplement, vol. 117, no. 2, pp. 393-404, 1996.

[9] N. Tyurina, V. Lipunov, and K. Victor, "MASTER prompt and follow-up GRB observations," Advances in Astronomy. In press.

[10] A. Panaitescu, "Prompt $\mathrm{GeV}$ emission in the synchrotron self-compton model for gamma-ray bursts," submitted, http://arxiv.org/abs/0811.1235v1.

[11] A. J. van der Horst, "GRB 081130B: Fermi GBM detection," GCN Circular, no. 8593, 2008.

[12] C. A. Wilson-Hodge, "GRB090305B: Fermi GBM detection," GCN Circular, no. 8972, 2009.
[13] P. N. Bhat, "GRB 090320B: Fermi GBM detection," GCN Circular, no. 9020, 2009.

[14] K. Ivanov, S. Yazev, E. Gorbovskoy, et al., "GRB 090328B: MASTER-Irkutsk prompt optical short burst observations," GCN Circular, no. 9065, 2009.

[15] V. Mangano, B. Sbarufatti, V. La Parola, and T. Ukwatta, "GRB 081102: Swift-XRT refined analysis,” GCN Circular, no. 8470, 2008.

[16] V. Lipunov, V. Kornilov, E. Gorbovskoy, et al., “GRB 081102: MASTER prompt optical limit," GCN Circular, no. 8471, 2008.

[17] E. Gorbovskoy, V. Lipunov, V. Kornilov, et al., "GRB 081102: MASTER refind and final results," GCN Circular, no. 8516, 2008.

[18] A. V. Zasov and K. A. Postnov, General Astrophysics, Vek 2, Fryazino, Russia, 2006.

[19] P. M. W. Kalberla, W. B. Burton, D. Hartmann, et al., "The Leiden/Argentine/Bonn (LAB) survey of Galactic HI. Final data release of the combined LDS and IAR surveys with improved stray-radiation corrections," Astronomy \& Astrophysics, vol. 440, no. 2, pp. 775-782, 2005.

[20] E. E. Fenimore, S. D. Barthelmy, W. H. Baumgartner, et al., "GRB 081102: Swift-BAT refined analysis," GCN Circular, no. 8468, 2008.

[21] P. Schady and J. K. Cannizzo, "Swift/UVOT observations of GRB 090424,” GCN Circular, no. 9234, 2009.

[22] E. Gorbovskoy, V. Lipunov, V. Kornilov, et al., "GRB 090424: MASTER-Net prompt optical limit," GCN Circular, no. 9252, 2009.

[23] E. Gorbovskoy, V. Lipunov, V. Kornilov, et al., "GRB 090424: MASTER-Net prompt optical observations," GCN Circular, no. $9233,2009$.

[24] T. Sakamoto, S. D. Barthelmy, W. H. Baumgartner, et al., "GRB 090424: Swift-BAT refined analysis," GCN Circular, no. 9231, 2009.

[25] V. Connaughton, "GRB 090424: Fermi GBM observation," GCN Circular, no. 9230, 2009.

[26] E. Gorbovskoy, V. Lipunov, V. Kornilov, et al., "GRB 081130; MASTER VWF prompt optical observations Fermi GRB," GCN Circular, no. 8585, 2009.

[27] E. Gorbovskoy, V. Lipunov, V. Kornilov, et al., "GRB 081130B: MASTER prompt optical observations," GCN Circular, no. 8597, 2008.

[28] E. Gorbovskoy, V. Lipunov, V. Kornilov, et al., "GRB090305B: MASTER-net prompt optical short burst observations," GCN Circular, no. 9004, 2009.

[29] E. Gorbovskoy, V. Lipunov, V. Kornilov, et al., "GRB 090320B: MASTER-Net prompt optical observations," GCN Circular, no. $9038,2009$.

[30] A. Goldstein, “GRB 090328B: Fermi GBM detection," GCN Circular, no. 9056, 2009.

[31] B. Paczynski, "Astronomy with small telescopes," The Publications of the Astronomical Society of the Pacific, vol. 118, no. 850, pp. 1621-1625, 2006. 

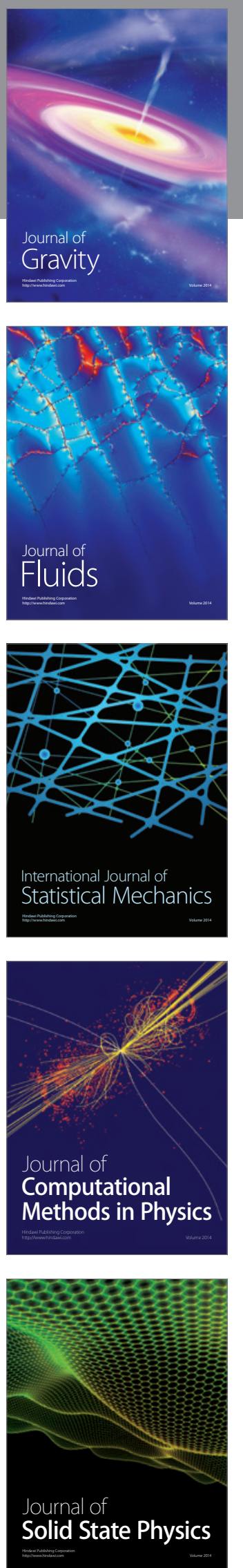

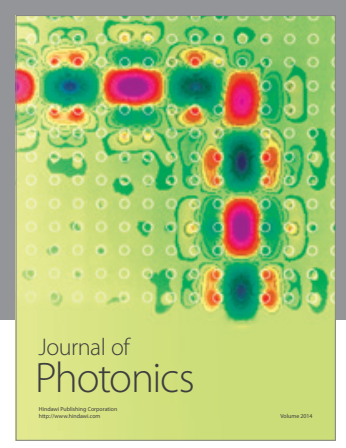

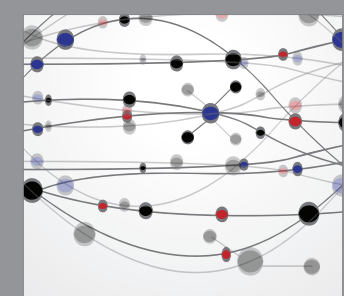

The Scientific World Journal
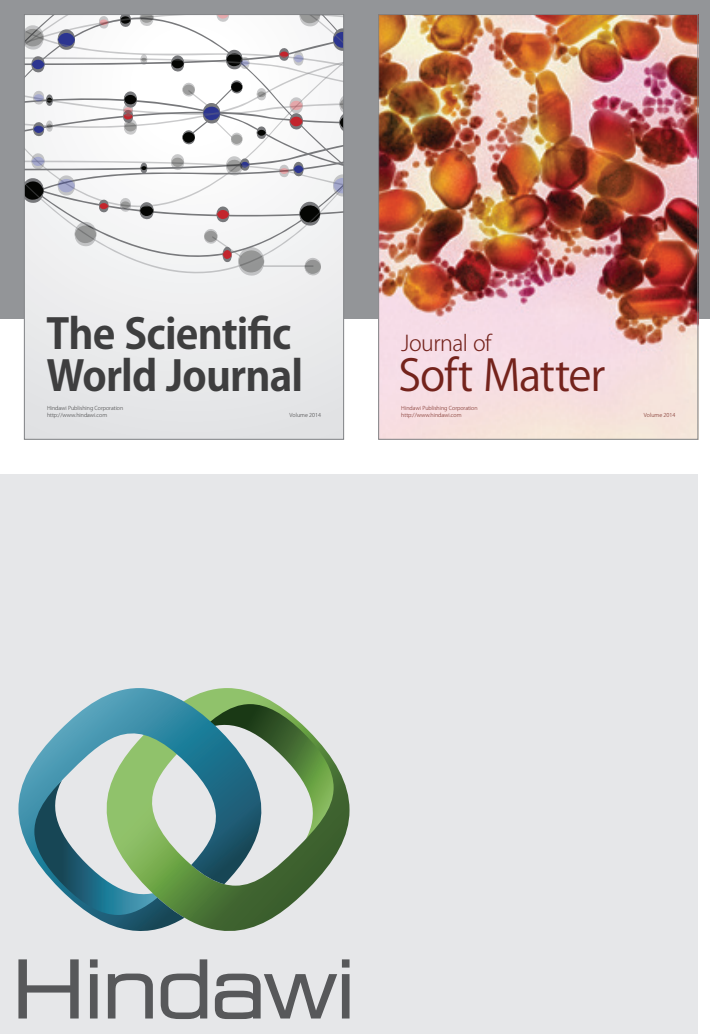

Submit your manuscripts at

http://www.hindawi.com
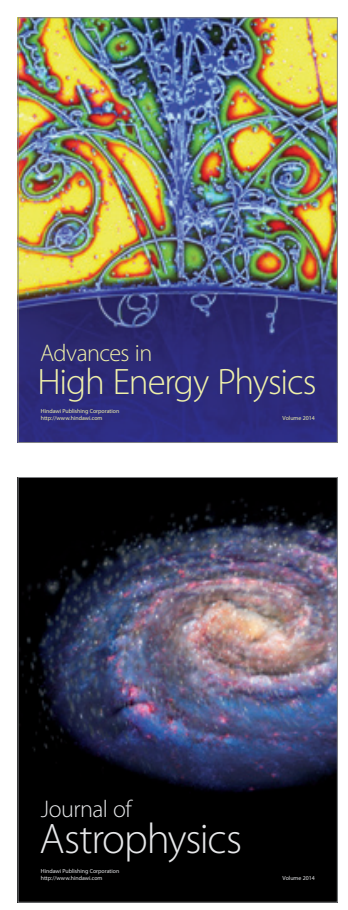
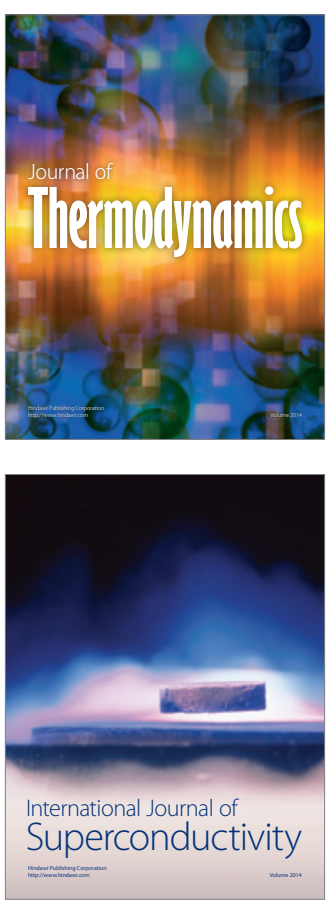
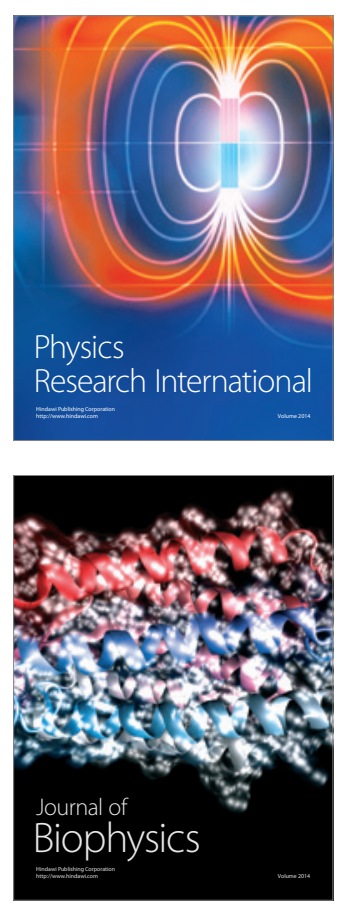
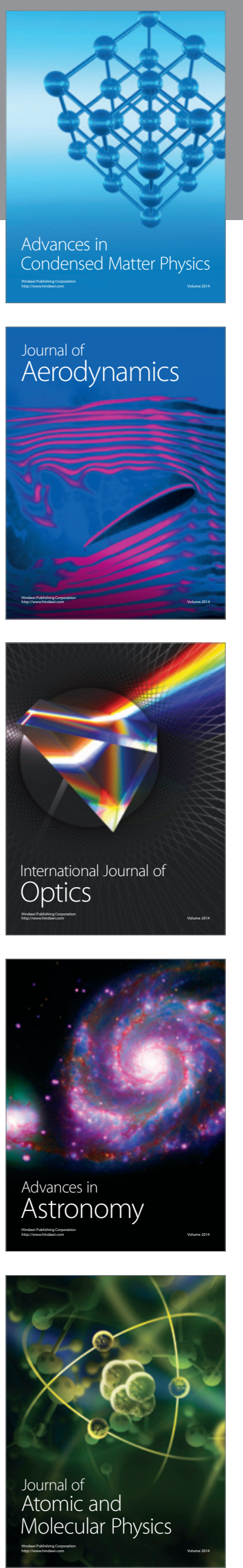